\title{
Régimen monetario e instituciones laborales: una interpretación alternativa de la tendencia a la disminución del traspaso del tipo de cambio (pass-through) en los países periféricos ${ }^{1}$
}

\author{
Martín Cherkasky y Martín Abeles
}

\section{Resumen}

En los últimos años, un conjunto de trabajos empíricos documentó la disminución de la intensidad del traspaso del tipo de cambio nominal a los precios internos en los países periféricos o en desarrollo. De conformidad con la hipótesis tradicional, esta tendencia - verificada en las dos últimas décadas - se interpretó como el resultado de una mayor credibilidad en la política monetaria, a raíz de la implementación de regímenes formales de metas de inflación. En este trabajo se presenta una interpretación alternativa, afín a la tradición estructuralista, que considera otras transformaciones registradas en paralelo a la instauración de los regímenes de metas de inflación como la flexibilización generalizada del mercado laboral. A partir de estimaciones empíricas para un conjunto de países periféricos relativas al período 1994-2016, se constató una marcada asociación estadística entre la intensidad del traspaso del tipo de cambio y un debilitamiento de las instituciones laborales.

\section{Palabras clave}

Tipo de cambio, política monetaria, empleo, mercado de trabajo, países en desarrollo Clasificación JEL

E02, E31, E58, J50

\section{Autores}

Martín Cherkasky es Asistente de Investigación en la oficina de la Comisión Económica para América Latina y el Caribe (CEPAL) en Buenos Aires. Correo electrónico: martin.cherkasky@cepal.org.

Martín Abeles es Director de la oficina de la CEPAL en Buenos Aires. Correo electrónico: martin.abeles@cepal.org.

Los autores agradecen los comentarios y las sugerencias de Francisco Arroyo Marioli, Pablo Bortz, Pablo Chena, Ariel Dvoskin, Germán Feldman, Gabriel Porcile, Juan Manuel Telechea, Fernando Toledo, Sebastián Valdecantos y Soledad Villafañe y la asistencia de Alicia Pita en la elaboración de las bases de datos. Los errores y las omisiones remanentes son de exclusiva responsabilidad de los autores. 


\section{Introducción}

Los economistas partían de una imperturbable fe en su ciencia como fuente de explicaciones verificables. La inflación se explicaba por factores que podían manipularse, es decir, por factores que podían modificarse sin necesidad de cambios en las autodefiniciones de la gente: el nivel de la demanda, el peso de los impuestos, la magnitud del déficit fiscal, el crecimiento de la oferta monetaria. Al comienzo de la década de 1980, estamos mejor preparados para preguntarnos si la inflación no está en gran medida incentivada por nuestras relaciones políticas, en otras palabras, en parte por las autodefiniciones implícitas en nuestras prácticas dominantes.

Charles Taylor ${ }^{2}$

Con la proliferación de los regímenes de metas de inflación en las últimas décadas, como por ejemplo el establecimiento de regímenes de tipo de cambio flexible, el análisis de la relación entre las fluctuaciones cambiarias y la inflación cobró una gran relevancia ${ }^{3}$. En los regímenes de metas de inflación es preciso que el traspaso del tipo de cambio a los precios internos (pass-through) sea lo más reducido posible, para evitar que la mayor volatilidad cambiaria, asociada a la libre flotación, dificulte el cumplimiento de un objetivo de inflación baja y estable. En los últimos años, en un conjunto de trabajos se detectó una tendencia a la disminución de los coeficientes del traspaso del tipo de cambio. Esto explica que las autoridades monetarias de muchos países hayan perdido el "miedo a flotar" en la implementación de la política monetaria 4 .

En la literatura especializada existen, a grandes rasgos, dos tipos de trabajos que estudian esta tendencia. El primero se centra en la disminución de la intensidad del traspaso sobre los precios de los productos de importación (Campa y Goldberg, 2005). De acuerdo con este conjunto de trabajos, uno de los principales motivos de la disminución en los coeficientes del traspaso observada obedece a un cambio en la composición de las importaciones a favor de productos cuyos precios son menos sensibles al tipo de cambio, dado el poder de mercado de las empresas importadoras y de la discriminación de precios en el mercado internacional. Según este enfoque, en el caso de los productos con mayor grado de diferenciación, es posible reducir el margen de ganancia de las empresas importadoras a fin de mantener su participación en el mercado 5 . Otro motivo expuesto en estos trabajos es la importancia creciente de los costos de los servicios no transables (cuyos precios

2 "Economists started off with an unshaken faith in their science as the source of verifiable explanations. Inflation was explained by factors that could be manipulated, that is, by factors which could be adjusted without any change in people's self-definitions: the level of demand, levels of taxation, size of government deficit, growth of money supply. At the beginning of the 1980s, we are more ready to ask ourselves whether inflation isn't largely fuelled by our political relations, in other words, in part by the self-definitions implicit in our dominant practices" (Taylor, 1985, pág. 115).

3 Los regímenes de metas de inflación tienen dos requisitos básicos: i) ausencia de dominancia fiscal y ii) ausencia de dominancia externa. Del primer requisito se deriva la recomendación de independencia del banco central con respecto a las autoridades políticas electas, que suele complementarse con reglas de comportamiento fiscal, como los límites legislativos a la monetización de los déficits fiscales. Del segundo requisito, que supone la no subordinación de la política monetaria a un objetivo de tipo de cambio (con miras a, por ejemplo, estimular las exportaciones), se deriva el establecimiento de regímenes de libre flotación del tipo de cambio. En la práctica, sin embargo, en los países donde se instrumentan regímenes formales de metas de inflación predominan los esquemas de flotación administrada, con intervenciones de la autoridad monetaria para acotar la volatilidad cambiaria a corto plazo, pero sin interferir, en teoría, con la determinación por el mercado del tipo de cambio real a largo plazo.

4 En la literatura se plantea que el miedo a la inflación explica el miedo a flotar de los bancos centrales de los países en los que el traspaso del tipo de cambio a los precios resulta elevado (Schönerwald da Silva y Vernengo, 2008). En los países con regímenes de flotación cambiaria, el miedo a la inflación se expresa mediante una respuesta de política más enérgica para acotar o contener las depreciaciones, en comparación con las apreciaciones. En América Latina, la respuesta de política asimétrica de los bancos centrales a los movimientos del tipo de cambio es más clara en países como el Brasil y México (Libman, 2018).

5 Esta explicación se basa en la teoría de fijación de precios según el mercado (pricing to market) (Dornbusch, 1987; Krugman, 1987; Marston, 1990) 
son menos sensibles al tipo de cambio) en el valor de los productos transables en los países en desarrollo, en la medida que aumenta su nivel de ingreso real (Frankel, Parsley y Wei, 2012).

Enel segundo tipo de trabajos se estudia la disminución del traspaso sobre los precios al consumidor en general (no solo los precios de importación) y se pone el acento en el papel que corresponde a las expectativas (Gagnon y Ihrig, 2004; Choudhri y Hakura, 2006; Carrière-Swallow y otros, 2016; Devereux y Yetman, 2010; Bailliu y Fujii, 2004). Estos trabajos se basan en la hipótesis de Taylor (2000), conforme a la cual, en un marco de fijación de precios escalonada en el tiempo y con competencia imperfecta, los incrementos de precios tienden a ser menores en un ambiente de baja inflación, donde se percibe que las variaciones en los costos son menos persistentes. En este contexto, la credibilidad de la autoridad monetaria tiene un papel central. En un régimen monetario en que la meta oficial de inflación constituye el ancla nominal de la economía, la credibilidad de los bancos centrales depende de la percepción de los agentes sobre la eficacia de la autoridad monetaria para cumplir con su objetivo (Choudhri y Hakura, 2001). Un banco central "creíble" permite que la meta oficial informe las expectativas inflacionarias de los agentes, independientemente de las fluctuaciones cambiarias. En ese sentido, en un conjunto de trabajos se ha mostrado la relevancia estadística de la credibilidad de la política monetaria a la hora de explicar la disminución del traspaso del tipo de cambio en los países en desarrollo en los últimos años (Carrière-Swallow y otros, 2016; Borensztein y Queijo von Heideken, 2016)6 ${ }^{6}$.

El traspaso de las variaciones del tipo de cambio nominal a los precios al consumidor puede descomponerse en dos rondas: el llamado efecto de la primera ronda, que hace referencia al impacto de las variaciones del tipo de cambio nominal sobre los precios de los bienes y servicios transables que, directa o indirectamente, forman parte de la canasta de consumo de los hogares; y el efecto de la segunda ronda, relativo al impacto de las variaciones del tipo de cambio nominal sobre el resto de los precios de bienes y servicios ${ }^{7}$. La intensidad del efecto de la segunda ronda depende de la capacidad (poder de mercado) de los proveedores de dichos bienes y servicios para recuperar la pérdida (directa o indirectamente) resultante de la inflación en bienes transables. El ajuste salarial puede representar una parte significativa de esa pérdida. El poder adquisitivo de los trabajadores suele verse muy afectado por las depreciaciones cambiarias. Suponiendo que existe resistencia salarial operativa, la intensidad de su efecto inflacionario (de segunda ronda) dependerá, en última instancia, de la capacidad de los empresarios de trasladar el aumento de los costos salariales a los precios de venta minoristas ${ }^{8}$.

Desde una perspectiva macroeconómica, existe una relación directa entre la intensidad del traslado de una modificación en el tipo de cambio nominal a los precios internos y la capacidad de resistencia salarial. Según el análisis convencional, esa capacidad de resistencia depende -al igual que la posibilidad de los empresarios de trasladar el aumento de los costos a los precios - del nivel de actividad económica, en el que la propia política monetaria puede cumplir un papel relevante. Asimismo, depende del grado de sindicalización existente y del tipo de instituciones laborales prevalecientes. Una menor intensidad en el traslado de las variaciones del tipo de cambio a los precios puede obedecer al debilitamiento sindical, la retracción de ciertas instituciones laborales o a una combinación de ambos fenómenos, más que a la mayor credibilidad de la autoridad monetaria. En este estudio se aporta evidencia empírica congruente con esta interpretación alternativa.

Esta hipótesis de trabajo es coherente con el enfoque estructuralista, en cuyo análisis de la inflación se atribuye un papel determinante a las instituciones que regulan el conflicto distributivo (Kalecki, 1971; Rowthorn, 1977; Frenkel, 1984; Noyola, 1956; Sunkel, 1958; Olivera, 1967; Vernengo, 2003), y

6 En el primero de los trabajos citados se utiliza el grado de acuerdo con las proyecciones de inflación de los analistas privados como variable sustitutiva de la credibilidad de la política monetaria, mientras en el segundo se considera la diferencia entre las expectativas de inflación (también de los analistas privados) y la meta del banco central.

7 En la primera ronda se incluye el efecto inflacionario de los bienes y servicios transables finales e intermedios incluidos en la producción local de bienes de consumo.

8 La resistencia salarial se define como la suba de los salarios nominales motivada por el objetivo de neutralizar, parcial o totalmente, la pérdida de poder adquisitivo generada por la inflación pasada. 
se relaciona con el debate acerca de la merma de la inflación en el período de la Gran Moderación (1987-2007), una etapa caracterizada por el debilitamiento del poder de negociación de los trabajadores $^{9}$. Esta perspectiva pone de relieve el tipo de arreglos institucionales necesarios para el establecimiento efectivo de regímenes de metas de inflación. Dado que trasciende la esfera estrictamente monetaria, esta perspectiva permite comprender mejor la relación entre la tendencia a la reducción de la inflación observada desde fines de la década de 1980, el debilitamiento de las instituciones laborales y la concomitante caída de la participación de los salarios en el ingreso verificada tanto en los países desarrollados como en desarrollo. La importancia que se atribuye a las instituciones laborales en este enfoque es común a otros marcos analíticos, incluidas la corriente poskeynesiana, la escuela francesa de la regulación y el enfoque denominado "variedades de capitalismo".

Este artículo se divide en seis secciones, incluida esta introducción. En la segunda sección se presenta un enfoque alternativo sobre la disminución del traspaso del tipo de cambio nominal en contraposición a las explicaciones convencionales. En la tercera sección se estima el traspaso del tipo de cambio en ventanas móviles para un panel de 22 países periféricos, a partir del método de proyección local de Jordà (2005), utilizado comúnmente en la literatura empírica (Carrière-Swallow y otros, 2016; Caselli y Roitman, 2016). En la cuarta sección se presentan distintas aproximaciones de operacionalización del poder de negociación de los trabajadores y se propone una estrategia de estimación de la resistencia salarial. En la quinta sección se evalúa estadísticamente la relevancia de las variables obtenidas en la etapa anterior en el traspaso total del tipo de cambio y por un conjunto de variables que suelen estar presentes en la literatura empírica. En la sexta sección se presentan las conclusiones.

\section{Traspaso del tipo de cambio, conflicto distributivo y resistencia salarial}

En este trabajo se adopta un enfoque alternativo para analizar la disminución del traspaso del tipo de cambio. En línea con el enfoque estructuralista, se considera a las instituciones del mercado de trabajo como el factor más importante en la regulación del conflicto distributivo. Con el fin de evaluar los efectos de las devaluaciones cambiarias sobre la distribución de los ingresos, se examinan dos relaciones estadísticas: i) una relación estática entre variables en niveles absolutos, a fin de mostrar los cambios distributivos que se derivan de una modificación del tipo de cambio real; y ii) una relación dinámica, para analizar el papel de la puja distributiva entre los actores locales y, en particular, de la resistencia salarial.

En primer lugar, se plantea una ecuación de precios desde el punto de vista de los $\operatorname{costos}^{10}$, en la que, según la formulación de Bastian y Setterfield (2017), los bienes de importación se incorporan desde el "enfoque del productor"11.

$$
P=(1+\tau)\left(W a+e P_{f} b\right)
$$

\footnotetext{
9 Perry y Cline (2016) brindan evidencia sobre el papel decisivo de la moderación de los salarios en la merma de la inflación en el período de la Gran Moderación en los Estados Unidos.

${ }^{10}$ La formulación de una expresión del nivel de precios desde el punto de vista de la presión sobre los costos se encuentra en línea con la teoría poskeynesiana, neokeynesiana (Carlin y Soskice, 2006) y estructuralista.

${ }^{11}$ Existen dos enfoques para modelar la inflación en una economía abierta (Bastian y Setterfield, 2017): i) el enfoque del consumidor, que trata las importaciones como bienes finales (Blecker, 1989 y 2011); y ii) el enfoque del productor, que trata las importaciones como bienes intermedios (Bhaduri y Marglin, 1990; Taylor, 2004).
} 
Donde $P$ es el precio del bien local, $W$ es el salario nominal, $e$ es el tipo de cambio nominal, $P_{f}$ es el precio de los bienes de importación en moneda extranjera, $a$ y $b$ son las relaciones de trabajo e importaciones, respectivamente, sobre el producto total, y $\tau$ es el margen de ganancia.

Luego se despeja convenientemente la ecuación (2.1) y se divide a ambos lados por $P$ para expresar los salarios y el tipo de cambio en términos reales.

$$
(1+\tau) W a=P-(1+\tau) e P_{f} b \Rightarrow(1+\tau) w a=1-(1+\tau) e^{R} b
$$

Donde $w$ es el salario real y $e^{R}$ es el tipo de cambio real.

Sobre la base de la ecuación (2.2) es posible mostrar los cambios distributivos que se derivan de una modificación del tipo de cambio real en dos casos extremos, suponiendo que los precios internacionales y la estructura productiva están dados:

i) Salario endógeno y tasa de ganancia exógena:

$$
\begin{aligned}
& w=\frac{1-(1+\tau) e^{R} b}{(1+\tau) a} \\
& \left.\frac{d w}{d e_{R}}\right|_{\tau=\bar{\tau}}=\frac{-b}{a}<0
\end{aligned}
$$

ii) Tasa de ganancia endógena y salario exógeno:

$$
\begin{gathered}
\tau=\frac{1-\left(w a+e_{R} b\right)}{w a+e_{R} b} \\
\left.\frac{d \tau}{d e_{R}}\right|_{w=\bar{w}}=\frac{-b}{\left(w a+e_{R} b\right)^{2}}<0
\end{gathered}
$$

Estos dos casos muestran que una modificación del tipo de cambio real tiene que ser absorbida por el salario real, por la tasa de ganancia o bien por una combinación de ambos, dados la estructura de costos y los precios internacionales de los productos de importación ${ }^{12}$. Este aspecto es importante para marcar la diferencia que existe entre la desinflación y la disminución del traspaso del tipo de cambio. Mientras que en el primer caso se puede producir en forma alineada una suba nominal cada vez menor de salarios, precios y tipo de cambio a partir de una mayor coordinación de expectativas (de manera que cada sector podría verse obligado a reducir sus aspiraciones de ingresos, pero sin que ello signifique una pérdida de ingresos reales con respecto al momento inicial) ${ }^{13}$, en el segundo caso, el menor traslado del tipo de cambio sobre los precios internos necesariamente implica un aumento de la pérdida de ingresos para al menos un sector local con respecto al momento previo a la devaluación (empresas, trabajadores o ambos).

\footnotetext{
${ }^{12}$ Al respecto, Dvoskin y Feldman (2015) estudian en detalle el vínculo negativo entre salario real y tipo de cambio real, dada una determinada tasa de beneficios, para distintas estructuras productivas de América Latina.

${ }^{13}$ En una situación ideal de consenso entre los trabajadores y las empresas sobre la base de un pacto social se podría reducir la inflación sin modificar la distribución funcional del ingreso (Cornwall 1990; Cornwall y Cornwall, 2001; Setterfield, 2006b).
} 
Esta distinción también es relevante para analizar el papel de la política macroeconómica: mientras en un proceso de desinflación la implementación de una meta podría contribuir a alinear las expectativas de los distintos actores, sin cambiar necesariamente la distribución de los ingresos, en un proceso de disminución del traspaso del tipo de cambio tiene que existir un elemento por el cual alguno de los sectores locales se vea obligado a aceptar una mayor pérdida de ingresos reales. En este marco, la credibilidad de los bancos centrales podría ser un motivo de la reducción del traspaso del tipo de cambio, en la medida en que la respuesta esperada de la autoridad monetaria ante una devaluación sea una política monetaria contractiva, que aumenta el desempleo y reduce la participación salarial. Otra alternativa es que la credibilidad de las medidas de desinflación se base en la posibilidad de reducir el poder de negociación de los trabajadores a partir de políticas de flexibilización laboral. Cornwall (1990) y Setterfield (2006b) definen esta última alternativa como "políticas de ingresos basadas en el miedo".

Para ilustrar la relación dinámica entre salarios y precios tras una devaluación, en un marco de conflicto distributivo, se presenta un sistema de dos ecuaciones. En primer lugar, se asume que la tasa de crecimiento de los salarios nominales pretendida por los sindicatos depende de dos elementos: la brecha entre el salario real deseado por los trabajadores y el salario real efectivo, y la inflación del período anterior (2.7a):

$$
\hat{w}_{t}=\mu_{1}\left(\omega_{w}-\omega_{t-1}\right)+\mu_{2} \hat{p}_{t-1}
$$

Donde $\hat{w}_{t}$ es la tasa de crecimiento de los salarios nominales, $\hat{p}_{t-1}$ es la inflación del período anterior, $\omega_{w}$ es el salario real objetivo de los trabajadores, $\mu_{1}$ es el parámetro que indica la medida en que los sindicatos reaccionan a la discrepancia entre el salario real objetivo y el efectivo, y $\mu_{2}$ es el parámetro que indica la medida en que los sindicatos pueden indexar los salarios sobre la base de la inflación pasada.

El primer componente refleja la brecha de aspiraciones en materia de ingresos de los trabajadores. Este componente es positivo en la medida en que los trabajadores no puedan alcanzar el salario que consideran justo, debido a una indexación parcial. El segundo término refleja el tamaño de la indexación, vale decir, la magnitud de la recuperación del salario real tras un aumento de los precios nominales, de conformidad con las especificaciones formuladas por Arestis y Sawyer (2005), Neville y Kriesler (2008) y Lavoie (2014), para quienes los sindicatos operan bajo expectativas adaptativas, a partir de la incertidumbre sobre el futuro ${ }^{14}$.

Los parámetros $\mu_{1}$ y $\mu_{2}$ representan el poder de negociación de los trabajadores y podrían expresarse en función de otras variables. En la expresión planteada por Rochon y Setterfield (2007) y Vera (2014), el poder de negociación de los trabajadores depende de la tasa de desempleo. Por otra parte, en la especificación utilizada por Setterfield (2006a) el poder de negociación de los trabajadores depende de los cambios institucionales en el mercado de trabajo.

En segundo lugar, se supone que la tasa de inflación se encuentra afectada por tres componentes: la brecha de aspiraciones en materia de ingresos, el costo salarial y el costo de los productos importados. El primer componente refleja que las empresas tienen un margen de ganancia objetivo sobre los costos unitarios de producción, que puede expresarse en términos del salario real ${ }^{15}$.

\footnotetext{
${ }^{14} \mathrm{Si}$ el segundo término de la ecuación (2.7.a) se viera reemplazado por un término que denota expectativa de inflación futura, esto no cambiaría el equilibrio de la inflación y del salario real a largo plazo, dado que este se encuentra gobernado por la brecha de aspiraciones en materia de ingresos. Esto es, si el ajuste de salarios reclamado por los trabajadores es menor que la inflación pasada (por basarse en una expectativa de inflación futura más baja), esta diferencia se verá reflejada en un mayor incremento del salario nominal en los períodos siguientes, debido a una mayor brecha entre el salario real deseado y el efectivo.

${ }^{15}$ Esta representación ha sido adoptada, entre otros autores, por Dutt (1987), Sawyer (1982), L. Taylor (1985 y 1991), Sarantis (1990), Smithin (1994), Cassetti (2003), Setterfield (2006a y 2009) y Godley y Lavoie (2007).
} 
El segundo componente de la ecuación de precios corresponde al costo salarial. Para simplificar el análisis se asume que la productividad del trabajo se mantiene constante y, por tanto, la dinámica del costo laboral depende solo de los salarios nominales ${ }^{16}$. Los componentes tercero y cuarto hacen referencia al efecto de los cambios en el tipo de cambio nominal y en los precios internacionales de los productos transables.

$$
\hat{p}_{t}=\varphi_{1}\left(\omega_{t-1}-\omega_{f}\right)+\varphi_{2} \hat{w}_{t}+\varphi_{3} \hat{e}_{t}+\varphi_{4 \hat{p}_{f}}
$$

Donde $\hat{e}$ es la variación del tipo de cambio nominal, $\varphi_{1}$ es el parámetro que indica la medida en que las empresas reaccionan a la discrepancia entre el salario efectivo y el objetivo, $\varphi_{2}$ es el parámetro que indica el efecto directo de los costos laborales en los precios, $\varphi_{3}$ es el parámetro que indica el efecto directo del tipo de cambio en la inflación, y $\varphi_{4}$ es el parámetro que indica el efecto directo del precio internacional de los productos transables.

Además, el salario real objetivo de los trabajadores y de las empresas se puede definir en función de un componente exógeno y del tipo de cambio real, en línea con Bastian y Setterfield (2017).

$$
\begin{aligned}
& \omega_{w}=\left[\omega_{w 0}+\mu_{2} e_{t-1}^{R}\right] \\
& \omega_{f}=\left[\omega_{f 0}-\varphi_{2} e_{t-1}^{R}\right]
\end{aligned}
$$

El objetivo de este trabajo consiste en evaluar el efecto de una devaluación en los precios y los salarios, que equivale a analizar una suba transitoria de la tasa de variación del tipo de cambio nominal sobre estas variables. Este choque se puede evaluar sobre dos tipos de equilibrio: un equilibrio de corto plazo y otro de largo plazo.

El equilibrio de corto plazo se puede definir como una posición en la que el impacto del choque se refleja en las tasas de variación en el corto plazo, sin que necesariamente se alcance una relación estable entre las variables. En el marco del sistema presentado anteriormente, este equilibrio se podría definir tras un período de un año, que es la frecuencia con la que se suelen negociar y ajustar los salarios.

El equilibrio a largo plazo se puede definir como una posición en la que las variables recobran una relación estable, luego de una $n$ cantidad de períodos. En el sistema presentado anteriormente, el mecanismo de atracción hacia el equilibrio a largo plazo es el término asociado a la brecha de aspiraciones en materia de ingresos. La ampliación de la brecha entre el salario real objetivo y el efectivo de los trabajadores determina que los salarios nominales crezcan por encima de los precios y del tipo de cambio nominal hasta que el salario real vuelva a alcanzar una posición estable. Si se deriva el salario real de equilibrio a partir de las ecuaciones anteriores y solo teniendo en cuenta la brecha de aspiraciones en materia de ingresos (véase el anexo A1), se puede mostrar que un cambio en la tasa de variación del tipo de cambio nominal no afecta el salario real de equilibrio de largo plazo, en la medida en que este cambio sea transitorio (2.11).

\footnotetext{
${ }^{16} \mathrm{Si}$ se consideraran los cambios en la productividad del trabajo, sería preciso expresar la brecha de aspiraciones en materia de ingresos según la participación de los salarios en el ingreso, de manera que los cambios en la productividad también formen parte de la disputa distributiva entre trabajadores y empresas.
} 


$$
\omega^{*}=\frac{\mu_{1} \omega_{w 0}+\varphi_{1} \omega_{f 0}+\left(\mu_{1} \mu_{2}-\varphi_{1} \varphi_{2}\right)\left[\frac{\left(\hat{e}+\hat{p}_{t}^{f}\right)\left(\mu_{1}+\varphi_{1}\right)-\mu_{1} \varphi_{1}\left(\omega_{w 0}-\omega_{f 0}\right)}{\mu_{1} \varphi_{1}\left(\mu_{2}+\varphi_{2}\right)}\right]}{\mu_{1}+\varphi_{1}}
$$

Si bien el equilibrio a largo plazo resulta útil para analizar una posición tendencial de las variables endógenas, constituye tan solo una posición ideal, en la que se asume que los parámetros son constantes y no están afectados por otros choques en la transición. Asimismo, en el sistema presentado no se consideran las interacciones entre la distribución y el empleo y el producto ni se incorporan consideraciones sobre el tipo de cambio real de equilibrio y su vínculo con la sostenibilidad de cuenta corriente.

A fin de simplificar este planteo para analizar un aumento transitorio del tipo de cambio nominal, se reformulan las expresiones $2.7 \mathrm{a}$ y $2.8 \mathrm{a}$ por $2.7 \mathrm{~b}$. y $2.8 \mathrm{~b}$, respectivamente, para representar un equilibrio de corto plazo, en el que se refleja el efecto de la devaluación en la inflación, pero donde las variables no necesariamente tienden a una relación estable. Para ello se reemplazan los términos asociados a la brecha de aspiraciones en materia de ingresos por una constante $\left(\alpha_{0}\right.$ y $\left.\beta_{0}\right)$, de forma de incorporar su nivel de partida. Esta formulación asume que la inflación solo está determinada por cambios nominales en los costos y permite centrarse en la relación dinámica entre precios y salarios.

$$
\begin{gathered}
\hat{w}_{t}=\alpha_{0}+\alpha_{1} \hat{p}_{t-1} \\
\hat{p}_{t}=\beta_{0}+\beta_{1} \hat{e}_{t}+\beta_{2} \hat{w}_{t}+\beta_{3} \hat{p}_{t}^{f}
\end{gathered}
$$

A continuación se reemplaza (2.7b) en (2.8b), se supone que en el equilibrio de corto plazo $\hat{p}_{t}=\hat{p}_{t-1}$ y se despeja $\hat{p}_{t}$.

$$
\hat{p}_{t}=\frac{\beta_{0}+\beta_{1} \hat{e}_{t}+\beta_{2} \alpha_{0}+\beta_{3 \hat{p}_{t}}^{f}}{\left(1-\alpha_{1} \beta_{2}\right)}
$$

Por último, se deriva con respecto a la variación del tipo de cambio para obtener una expresión del traspaso del tipo de cambio.

$$
\frac{d \hat{p}_{t}}{d \hat{e}_{t}}=\beta_{1} \frac{1}{\left(1-\alpha_{1} \beta_{2}\right)}
$$

En la expresión (2.13) se pueden distinguir dos componentes. El primero se asocia al efecto directo (o de primera ronda) y está representado por el parámetro $\beta_{1}$. Este efecto indica la medida en que los movimientos del tipo de cambio se trasladan a los precios al consumidor a partir de cambios en el precio de los productos transables. El segundo componente se asocia al efecto indirecto (o de segunda ronda). Este efecto depende de la resistencia salarial $\left(\alpha_{1}\right)$ y de la incidencia de los salarios en el costo unitario de producción $\left(\beta_{2}\right)^{17}$. Desde una perspectiva estructuralista, el efecto directo de la devaluación en los precios internos puede presentarse como el choque que impulsa la inflación por encima de su equilibrio inicial, y los efectos de segunda ronda -asociados principalmente a la negociación salarial - pueden presentarse como los que la propagan (Noyola, 1956; Furtado, 1963).

\footnotetext{
17 Cabe destacar que si la incidencia de los salarios en el costo unitario de producción aumentara (y la contracara fuera una reducción de la incidencia de las importaciones en igual magnitud), y considerando un traspaso del precio de los productos de importación a los precios al consumidor mayor a la resistencia salarial, el traspaso total del tipo de cambio sobre los precios al consumidor debería reducirse.
} 
La hipótesis de este trabajo es que la disminución del traspaso del tipo de cambio de las últimas décadas se asocia principalmente a una menor resistencia salarial, explicada por el menor poder de negociación de los trabajadores, tanto por el debilitamiento de las instituciones laborales, como por los mecanismos que presionan sobre el desempleo. Entre estos últimos se incluye la política que el banco central puede llevar a cabo con el objetivo de alcanzar su meta inflacionaria.

Para evaluar empíricamente la relevancia de la resistencia salarial en la determinación del traspaso del tipo de cambio sobre los precios, el siguiente razonamiento sigue tres etapas: i) se estima el traspaso del tipo de cambio en el conjunto de países considerados, ii) se estima la resistencia salarial, y iii) se realiza una regresión de los coeficientes de traspaso del tipo de cambio, en la que se incluyen como variables explicativas los coeficientes de resistencia salarial y un conjunto de variables de control utilizadas comúnmente en la literatura.

\section{Evidencia sobre la disminución del traspaso del tipo de cambio}

\section{Estimación del traspaso total del tipo de cambio}

Para obtener la respuesta de los precios internos acumulada a un año se realiza la estimación del traspaso total del tipo de cambio a partir del método de proyección local de Jordà (2005). Este método fue utilizado en otros trabajos recientes que miden el traspaso del tipo de cambio (Carrière-Swallow y otros, 2016; Caselli y Roitman, 2016). La especificación estimada es la siguiente:

$$
\begin{aligned}
& \operatorname{lnp}_{i, t+h-1}-\ln p_{i, t-1}=\beta_{0}+\sum_{j=0}^{j}\left(\beta_{1, j}^{h} \operatorname{TCNE}_{i, t-j}\right)+\beta_{2}^{h} \text { energia }_{t}+ \\
& \beta_{3}^{h} \text { alimentos }_{t}+\beta_{4, j}^{h} p_{i, t-1}+\beta_{5, j}^{h} \text { brecha }_{i, t}+\mu_{i, t}^{h}+\epsilon_{i, t}^{h}
\end{aligned}
$$

Donde $p$ es el índice de precios al consumidor, TCNE es la diferencia logarítmica del tipo de cambio nominal efectivo, energía y alimentos son las diferencias logarítmicas del precio internacional de la energía y de los alimentos, respectivamente, brecha es la brecha del producto, $\mu$ es un efecto fijo por país, $\epsilon$ es un término de error, $h$ es el horizonte de proyección, $i$ cada uno de los países incluidos en el panel y $j$ la cantidad de rezagos. La cantidad de rezagos considerada para el tipo de cambio es de uno $(j=1)$ y el horizonte de proyección elegido es de cuatro trimestres $(h=4)$. La elección de este horizonte temporal (un año) se encuentra en línea con la frecuencia de negociación y ajuste de los salarios, fundamental para tomar en consideración los efectos de segunda ronda de la variación del tipo de cambio. El parámetro de interés es $\beta_{1, j=0}^{h=4}$, que representa el traspaso del tipo de cambio sobre los precios al consumidor acumulado a un año.

El método de proyección local estima el efecto de la variable explicativa en " $t$ " sobre la variable explicada en " $t+h$ ", donde " $h$ " es el horizonte de proyección elegido. En este caso, la variable explicada se expresa como la variación acumulada. La principal ventaja del método de proyección local frente a la estimación de un modelo de vectores autorregresivos (VAR) es que es menos sensible a errores de especificación ante el incremento del número de regresores, rezagos y del horizonte de proyección (Jordà, 2005; Teulings y Zubanov, 2014). La desventaja es que el término de error tiene, por construcción, una forma de media móvil que introduce correlación serial e impide la inferencia estadística estándar. Para controlar por correlación serial se adoptan los errores estándar de Driscoll-Kraay para las estimaciones ${ }^{18}$.

18 Driscoll y Kraay (1998). 
Para la especificación se considera el tipo de cambio nominal efectivo (TCNE), un indicador multidivisa que abarca de manera más amplia el conjunto de los precios relativos que afectan el índice de precios al consumidor (IPC). La consideración del TCNE es más relevante en el caso de los países con un menor vínculo comercial con los Estados Unidos y cuyos socios comerciales tienen monedas con una relación más inestable frente al dólar estadounidense. En estos casos, la divergencia entre el TCNE y el tipo de cambio nominal bilateral (TCNB) tiende a ser mayor ${ }^{19}$. Mediante la incorporación de los precios internacionales de los alimentos y de la energía se busca controlar el componente importado del proceso inflacionario, mientras que con la inflación del período anterior se capta la inercia inflacionaria. La inclusión de la brecha del producto permite controlar por presiones inflacionarias generadas por excesos de demanda, en línea con el enfoque convencional. Desde una perspectiva alternativa, esta variable podría permitir controlar por la intensidad de la puja distributiva, ante la imposibilidad de incorporar los costos laborales unitarios y la tasa de desempleo, por la menor disponibilidad de datos. En este trabajo se aproxima a partir del componente cíclico del producto, obtenido mediante la aplicación del filtro de Hodrick-Prescott. Por último, se incluyen efectos fijos por país para controlar por características estructurales de cada uno de ellos.

El período muestral va de 1994 a 2016 y la frecuencia de los datos es trimestral. El panel incluye 22 países que pertenecen en su mayoría a América Latina y Europa Oriental (véase el cuadro 1). En 15 de los países examinados se adoptaron regímenes de metas de inflación ${ }^{20}$.

Cuadro 1

Países considerados

\begin{tabular}{llll}
\hline Argentina & Croacia & Malasia & Sudáfrica \\
\hline Bolivia (Estado Plurinacional de) & Federación de Rusia & México & Tailandia \\
\hline Brasil & Filipinas & Paraguay & Turquía \\
\hline Bulgaria & Hungría & Perú & Uruguay \\
\hline Chile & India & Polonia & \\
\hline Colombia & Indonesia & Rumania & \\
\hline
\end{tabular}

Fuente: Elaboración propia.

Nota: Se considera la clasificación de países emergentes y en desarrollo del Fondo Monetario Internacional (2018).

La muestra de datos incluye episodios de fuerte devaluación y períodos de hiperinflación (véase el cuadro 2). Las observaciones en las que las variaciones trimestrales del TCNE y del IPC son superiores al 100\% fueron excluidas de la muestra, junto con las tres observaciones subsiguientes que afectan la inflación acumulada a un año. Si bien es poco probable que estas observaciones se refieran a un funcionamiento normal de las instituciones de negociación salarial y fijación de precios, su inclusión como parte de la muestra tiende a distorsionar las estimaciones. Este tratamiento de los datos es similar al realizado por Caselli y Roitman (2016) y Fischer, Sahay y Vegh (2002).

\footnotetext{
${ }^{19}$ En trabajos como los de Carrière-Swallow y otros (2016) y Caselli y Roitman (2016) también se utiliza el tipo de cambio nominal efectivo para la estimación del traspaso del tipo de cambio.

${ }^{20}$ En 13 de esos 15 países el régimen de metas de inflación funcionó durante más de la mitad de la última década y media (2001-2016). Estos países son: Brasil, Chile, Colombia, Filipinas, Hungría, Indonesia, México, Perú, Polonia, Rumania, Sudáfrica, Tailandia y Turquía. Mientras en la India se adoptó en 2016, en la Argentina se adoptó formalmente en 2017 y se abandonó en 2018.
} 


\section{Cuadro 2}

Estadísticas descriptivas del panel de datos

\begin{tabular}{lccccc}
\hline Variable & Observaciones & Promedio & Desviación estándar & Mínimo & Máximo \\
\hline$\Delta \mathrm{nnIPC}$ & 2318 & 0,040 & 0,105 & $-0,038$ & 2,144 \\
\hline$\Delta \mathrm{n}$ TCNE & 2334 & 0,028 & 0,121 & $-0,379$ & 1,753 \\
\hline$\Delta \mathrm{n} \mathrm{nal}$ & 2266 & 0,003 & 0,059 & $-0,304$ & 0,163 \\
\hline$\Delta \mathrm{n} \mathrm{nPer}$ & 2178 & 0,010 & 0,129 & $-0,627$ & 0,262 \\
\hline $\ln \left(\mathrm{y} / \mathrm{y}^{\star}\right)$ & 1935 & 1,010 & 0,033 & 0,728 & 1,248 \\
\hline
\end{tabular}

Fuente: Elaboración propia, sobre la base de datos del Fondo Monetario Internacional (FMI), la Comisión Económica para América Latina y el Caribe (CEPAL), Bruegel y fuentes nacionales.

Nota: $\ln \left(y / y^{\star}\right)$ es el componente cíclico del producto, a partir del cual se aproxima la brecha.

Las estimaciones realizadas en ventanas móviles de 12 años confirman los resultados encontrados en la literatura, con una disminución del traspaso del tipo de cambio a los precios al consumidor de 0,52 en el período 1994-2005 a 0,16 en el período 2005-2016 (véase el gráfico 1). Las estimaciones para distintos horizontes temporales también muestran que la mayor disminución del traspaso del tipo de cambio se concentra entre el segundo y el tercer trimestre posterior a una devaluación y que el efecto del tipo de cambio se estabiliza entre el tercer y el cuarto trimestre (véase el gráfico 2). Esto indicaría la conveniencia de tomar un horizonte de cuatro trimestres para medir el efecto total.

\section{Gráfico 1}

Traspaso del tipo de cambio en países periféricos en ventanas móviles

(En porcentajes)

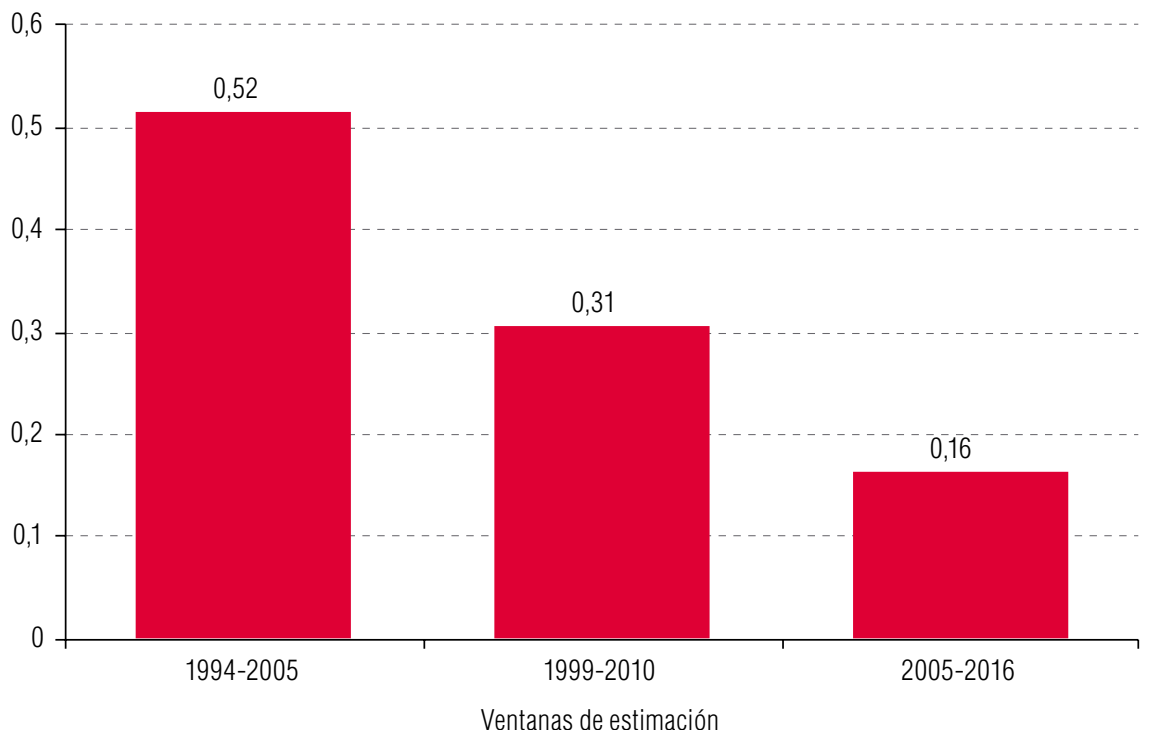

Fuente: Elaboración propia.

Nota: Respuesta acumulada de los precios al consumidor un año después de un aumento del $1 \%$ del tipo de cambio nominal efectivo. En las estimaciones se considera a los siguientes países: Argentina, Bolivia (Estado Plurinacional de), Brasil, Bulgaria, Chile, Colombia, Croacia, Federación de Rusia, Filipinas, Hungría, India, Indonesia, Malasia, México, Paraguay, Perú, Polonia, Rumania, Sudáfrica, Tailandia, Turquía y Uruguay. 


\section{Gráfico 2}

Traspaso del tipo de cambio en países periféricos para distintos horizontes temporales

(En porcentajes)

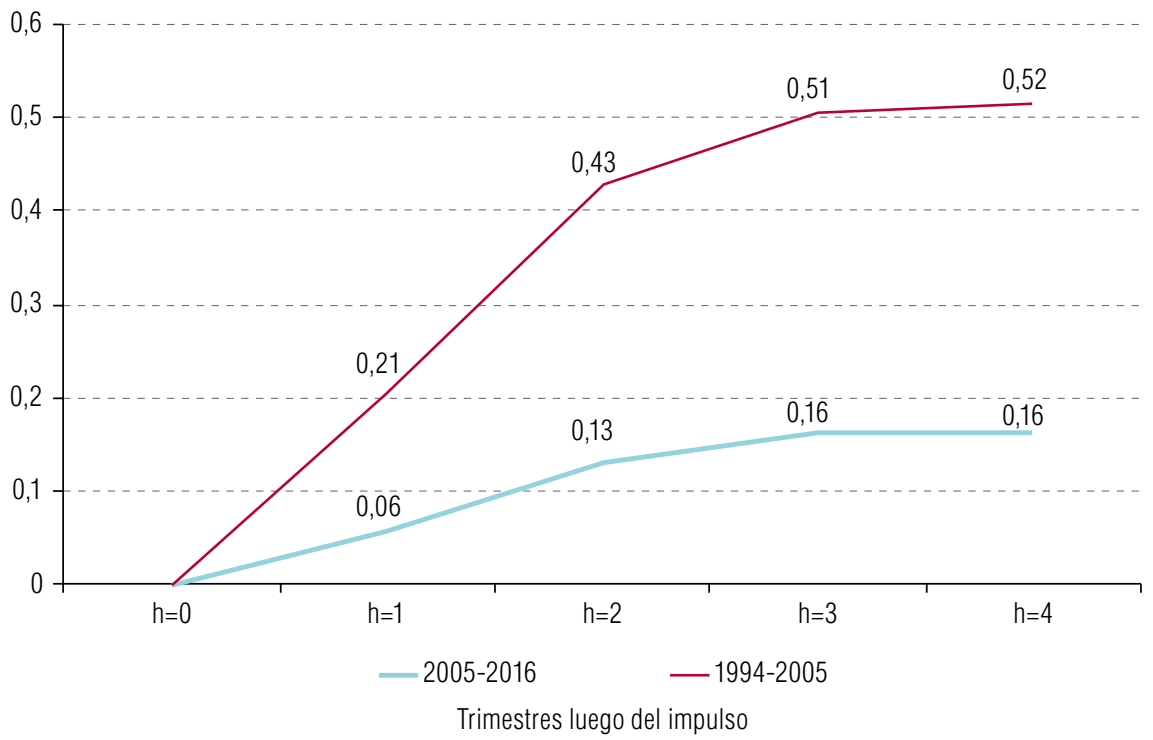

Fuente: Elaboración propia.

Nota: Respuesta acumulada de los precios al consumidor uno, dos, tres y cuatro trimestres después de un aumento del 1\% del tipo de cambio nominal efectivo. En las estimaciones se considera a los siguientes países: Argentina, Bolivia (Estado Plurinacional de), Brasil, Bulgaria, Chile, Colombia, Croacia, Federación de Rusia, Filipinas, Hungría, India, Indonesia, Malasia, México, Paraguay, Perú, Polonia, Rumania, Sudáfrica, Tailandia, Turquía y Uruguay.

Las estimaciones por regiones muestran que la disminución del traspaso del tipo de cambio fue generalizada. La mayor disminución del traspaso del tipo de cambio, de 0,86 en el período 1994-2005 a 0,17 en el período 2005-2016, se registró en Europa Oriental, mientras que lo hizo en forma menos pronunciada en América Latina, pasando del 0,34 al 0,18. Los países considerados de Asia son los que registraron un menor traspaso del tipo de cambio en los últimos años (véase el gráfico 3).

Con el objetivo de indagar en qué medida esta reducción del traspaso del tipo de cambio puede asociarse a la mayor credibilidad de la política monetaria se realizaron estimaciones para dos grupos de países: aquellos donde se adoptaron regímenes de metas de inflación por un período prolongado ${ }^{21}$ y aquellos donde no se implementaron o se implementaron recientemente. Los resultados obtenidos revelan dos cosas. Por una parte, que la reducción del traspaso del tipo de cambio se verifica indistintamente en países con y sin regímenes de metas de inflación. Esto podría evidenciar que la reducción del traspaso del tipo de cambio obedece a un proceso más amplio, de carácter global, no vinculado necesariamente a la mayor credibilidad de la política monetaria. Por otra parte, las estimaciones muestran que la reducción del traspaso del tipo de cambio fue mayor en los países donde se implementaron regímenes de metas de inflación (véase el gráfico 4). Si bien este resultado indica que la implementación de regímenes de metas de inflación puede haber contribuido a reducir el traspaso del tipo de cambio con mayor intensidad, también podría indicar que otros posibles factores determinantes de esa reducción (como una mayor apertura comercial o una menor regulación laboral) se dieron con mayor intensidad en este grupo de países.

${ }^{21}$ Se trata de los países en los que el régimen de metas de inflación estuvo vigente por más de la mitad de la última ventana de tiempo (2005-2016), es decir, donde fue implementado en 2010 o con anterioridad. Estos son: Brasil, Chile, Colombia, Filipinas, Hungría, Indonesia, México, Perú, Polonia, Rumania, Sudáfrica, Tailandia y Turquía. 


\section{Gráfico 3}

Traspaso del tipo de cambio por regiones

(En porcentajes)

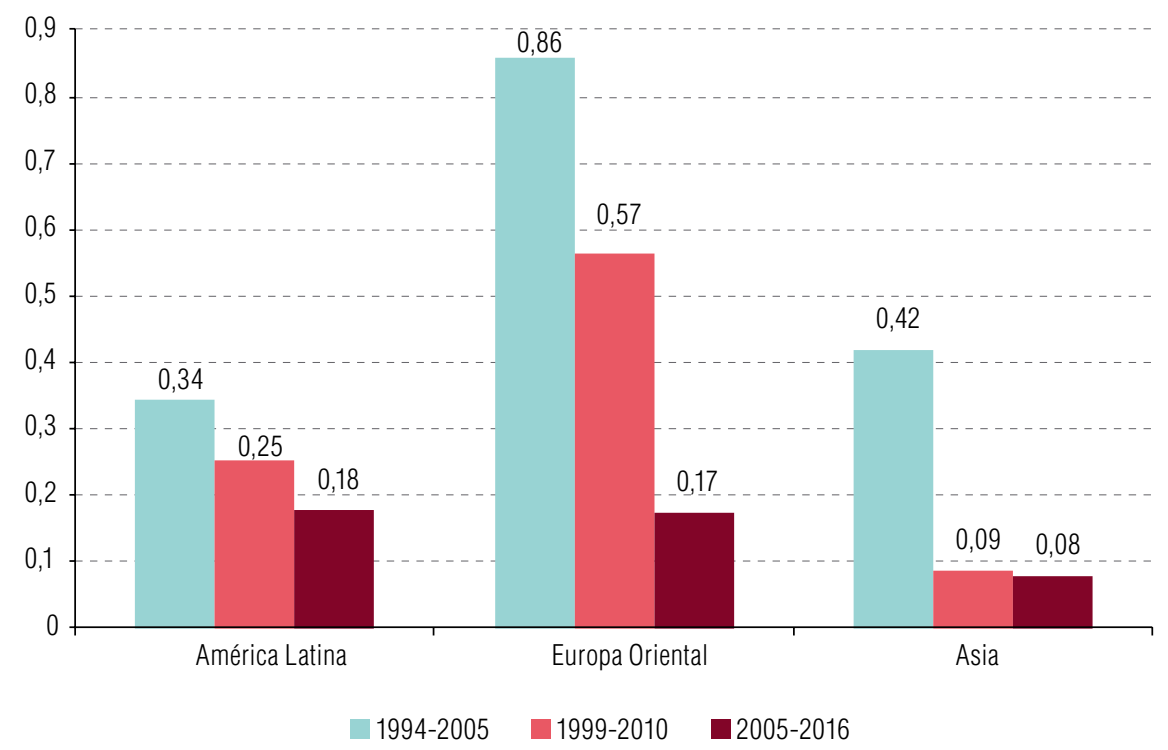

Fuente: Elaboración propia.

Nota: Respuesta acumulada de los precios al consumidor un año después de un aumento del $1 \%$ del tipo de cambio nominal efectivo. América Latina incluye: Argentina, Bolivia (Estado Plurinacional de), Brasil, Chile, Colombia, México, Paraguay, Perú y Uruguay; Europa Oriental incluye: Bulgaria, Croacia, Federación de Rusia, Hungría, Polonia, Rumania y Turquía; Asia incluye: Filipinas, India, Indonesia, Malasia y Tailandia.

\section{Gráfico 4}

Traspaso del tipo de cambio por regímenes monetarios en países periféricos (En porcentajes)

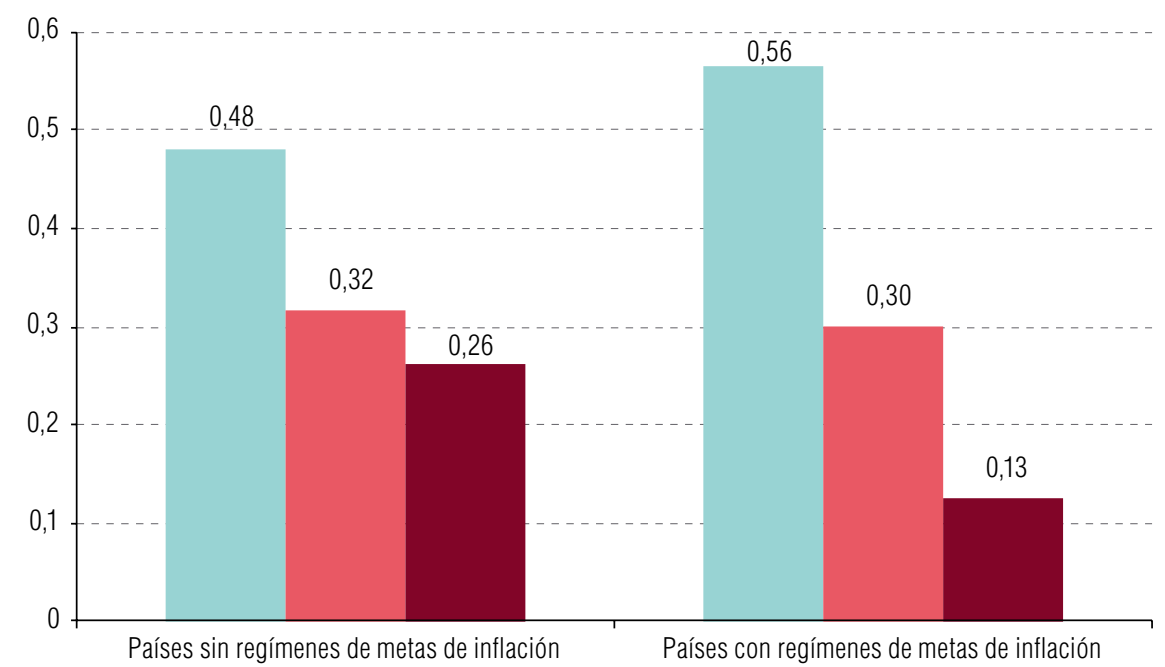

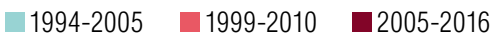

Fuente: Elaboración propia.

Nota: Respuesta acumulada de los precios al consumidor un año después de un aumento del $1 \%$ del tipo de cambio nominal efectivo. En los países con regímenes de metas de inflación (implementados en 2010 o con anterioridad) se incluye a: Brasil, Chile, Colombia, Filipinas, Hungría, Indonesia, México, Perú, Polonia, Rumania, Sudáfrica, Tailandia y Turquía. En los países sin regímenes de metas de inflación (o implementados desde 2011) se incluye a: Argentina, Bolivia (Estado Plurinacional de), Bulgaria, Croacia, Federación de Rusia, India, Malasia, Paraguay y Uruguay. 


\section{Medición del efecto de primera ronda}

Una vez estimado el traspaso total del tipo de cambio cabe preguntarse en qué medida la disminución observada en las últimas décadas respondió al efecto de primera ronda, vinculado a los precios de los productos transables, y en qué medida obedeció al efecto de segunda ronda, vinculado a los no transables. Para ello es necesario estimar el efecto de primera ronda por separado. Un indicador de referencia utilizado en la literatura para el efecto de primera ronda es la participación de los productos importados en la canasta de consumo de los hogares 22 .

La medición del efecto de primera ronda de referencia se realiza a partir de matrices de insumo-producto (Burstein, Eichenbaum y Rebelo, 2005; Carrière-Swallow y otros, 2016; Gopinath, 2015), siguiendo el cálculo realizado por Carrière-Swallow y otros (2016), a partir de las matrices de insumo-producto multirregionales de la base de datos Eora (Lenzen y otros, 2012 y 2013).

El componente de importaciones finales se calcula como el cociente entre las importaciones de bienes para el consumo y el consumo total. El componente importado intermedio se calcula multiplicando el producto sectorial local destinado al consumo privado y la participación de las importaciones intermedias de cada sector. Luego se realiza la suma para todos los sectores y se divide por el consumo privado total.

Se observa que el componente importado del consumo privado aumentó en los países de América Latina y Europa Oriental incluidos en el panel (véase el gráfico 5). Estos resultados se encuentran en línea con la gravitación cada vez mayor de las cadenas globales de valor en los países considerados, que incrementó los flujos de comercio en ambos sentidos. En el caso de los países asiáticos tendió a mantenerse estable.

En el gráfico 6 puede observarse que la disminución del traspaso total del tipo de cambio de los últimos años contrasta con el aumento de la participación de los productos importados. En América Latina y Europa Oriental, los coeficientes de traslado del tipo de cambio tienden a acercarse al componente importado del consumo de los hogares. Estos datos no brindan evidencia suficiente para mostrar que el efecto de primera ronda no se redujo en estos países, pero sugieren que la disminución del traspaso del tipo de cambio de los últimos años se encuentra más fuertemente vinculada a los efectos de segunda ronda asociados a la dinámica de los precios de los bienes y servicios no transables. Con el fin de analizar en qué medida el debilitamiento de las instituciones laborales incidió en el menor efecto de segunda ronda, en la siguiente sección se estima una variable sustitutiva de la resistencia salarial.

\footnotetext{
22 Este valor de referencia supone que el traspaso del tipo de cambio sobre el precio de los bienes y servicios importados es completo y excluye a los bienes de exportación que forman parte de la canasta de consumo de los hogares. Si bien la evidencia empírica muestra que el traspaso del tipo de cambio sobre los productos importados tiende a ser completo, en algunos casos puede ser menor y es más usual en el caso de los productos con mayor grado de diferenciación (Campa y Goldberg, 2005).
} 


\section{Gráfico 5}

Componente importado del consumo privado por regiones

(En porcentajes)

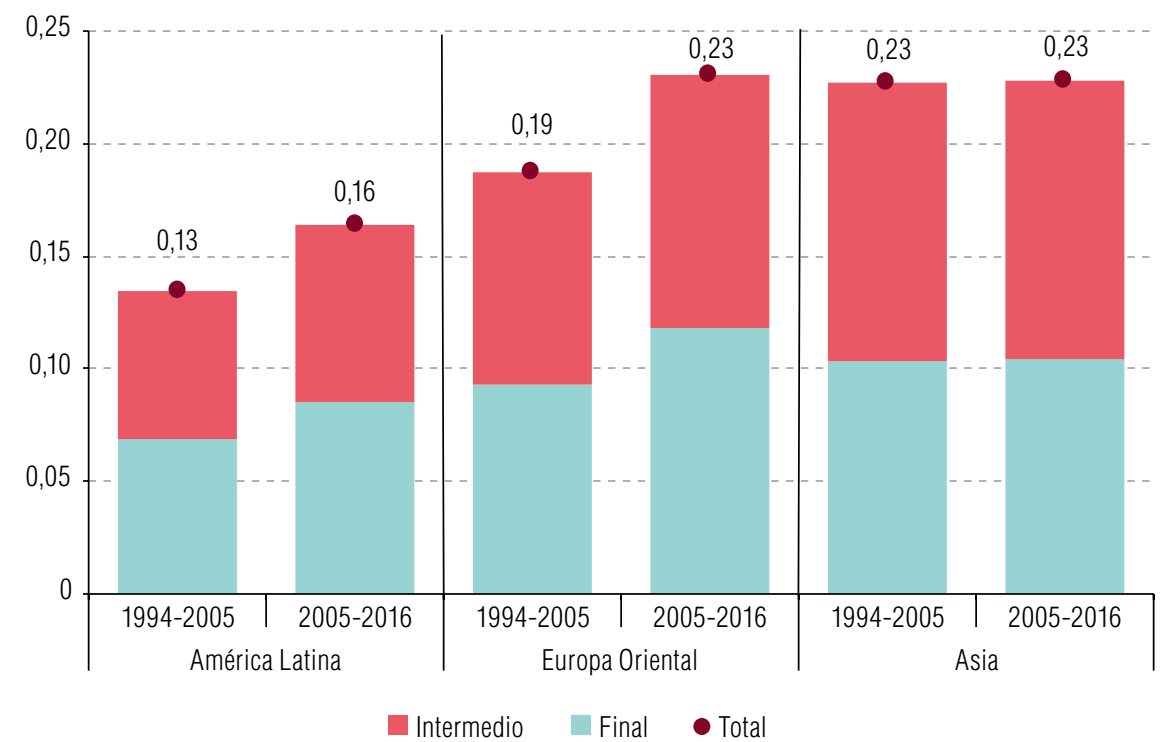

Fuente: Elaboración propia, sobre la base de M. Lenzen y otros, "Building EORA: a global multi-region input-output database at high country and sector resolution", Economic Systems Research, vol. 25, № 1, Abingdon, Taylor \& Francis, 2013, y "Mapping the structure of the world economy", Environmental Science \& Technology, vol. 46, № 15, Washington, D.C., American Chemical Society, 2012.

Nota: El gráfico muestra la participación de las importaciones intermedias, finales y totales en el consumo privado. América Latina incluye: Argentina, Bolivia (Estado Plurinacional de), Brasil, Chile, Colombia, México, Paraguay, Perú y Uruguay; Europa Oriental incluye: Bulgaria, Croacia, Federación de Rusia, Hungría, Polonia, Rumania y Turquía; Asia incluye: Filipinas, India, Indonesia, Malasia y Tailandia.

\section{Gráfico 6}

Traspaso del tipo de cambio y componente importado del consumo privado por regiones (En porcentajes)

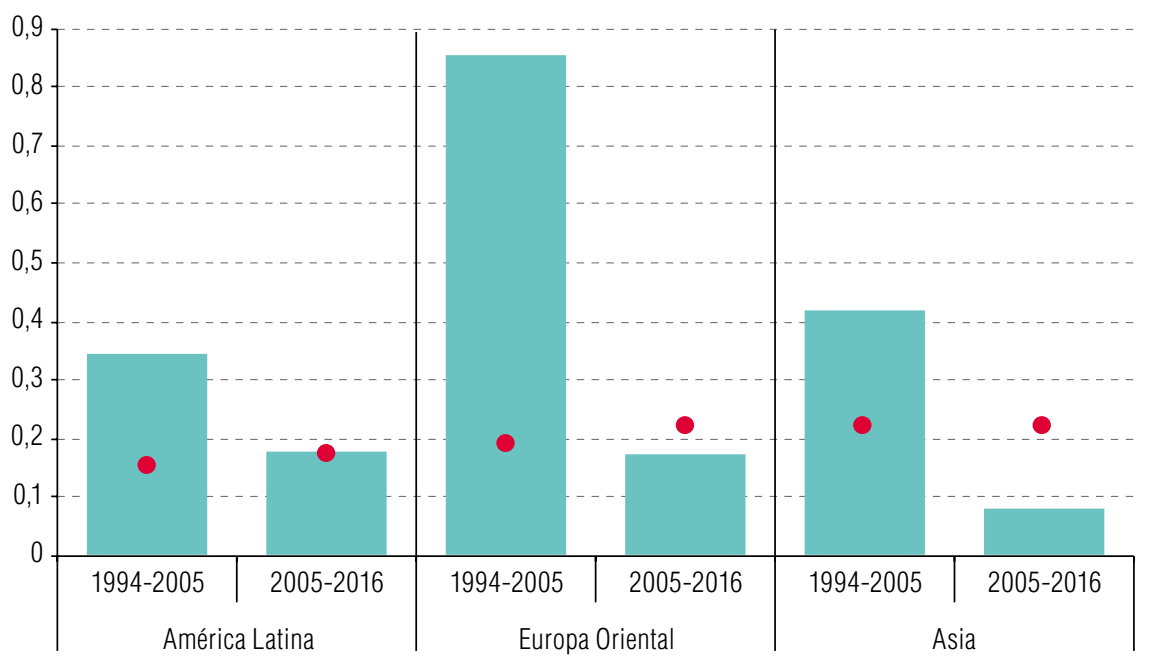

Traslado del tipo de cambio

- Componente importado del consumo privado

Fuente: Elaboración propia.

Nota: Las barras sólidas muestran la respuesta acumulada de los precios al consumidor un año después de un aumento del $1 \%$ del tipo de cambio nominal efectivo. Los marcadores muestran la participación de las importaciones (intermedias y finales) en el consumo privado. América Latina incluye: Argentina, Bolivia (Estado Plurinacional de), Brasil, Chile, Colombia, México, Paraguay, Perú y Uruguay; Europa Oriental incluye: Bulgaria, Croacia, Federación de Rusia, Hungría, Polonia, Rumania y Turquía; Asia incluye: Filipinas, India, Indonesia, Malasia y Tailandia. 


\section{Las instituciones laborales y la resistencia salarial}

En las últimas décadas, las instituciones laborales tendieron a debilitarse a nivel mundial (Berg, 2015). El aumento de la fuerza de trabajo a escala global, vinculado con el ingreso de China y la India - pero también de la Federación de Rusia y otros países del exbloque soviético - al mercado mundial, presionaron sobre los niveles de desempleo y favorecieron un proceso de flexibilización laboral en prácticamente todos países, dando lugar a una intensificación de la disciplina salarial a escala planetaria (Freeman, 2006).

La mayor flexibilidad laboral se manifiesta de distintas maneras, incluidas la proliferación de empleos menos estables (a plazo fijo o con un mayor período de prueba), la reducción de la seguridad laboral (con acortamiento del período de preaviso y reducción de las indemnizaciones por despidos), la descentralización de la negociación colectiva (con eliminación o suspensión de los convenios colectivos nacionales), el debilitamiento e intervención de la negociación colectiva (con suspensión de los contratos vigentes y limitación de la duración de los contratos) y el debilitamiento de los sindicatos (que facilita las condiciones para alcanzar acuerdos con representantes del personal no sindicado) (Naciones Unidas, 2016, pág. 10). En el gráfico 7 puede observarse la tendencia hacia la reducción de la cobertura de las negociaciones colectivas en un conjunto de países de la Organización de Cooperación y Desarrollo Económicos (OCDE).

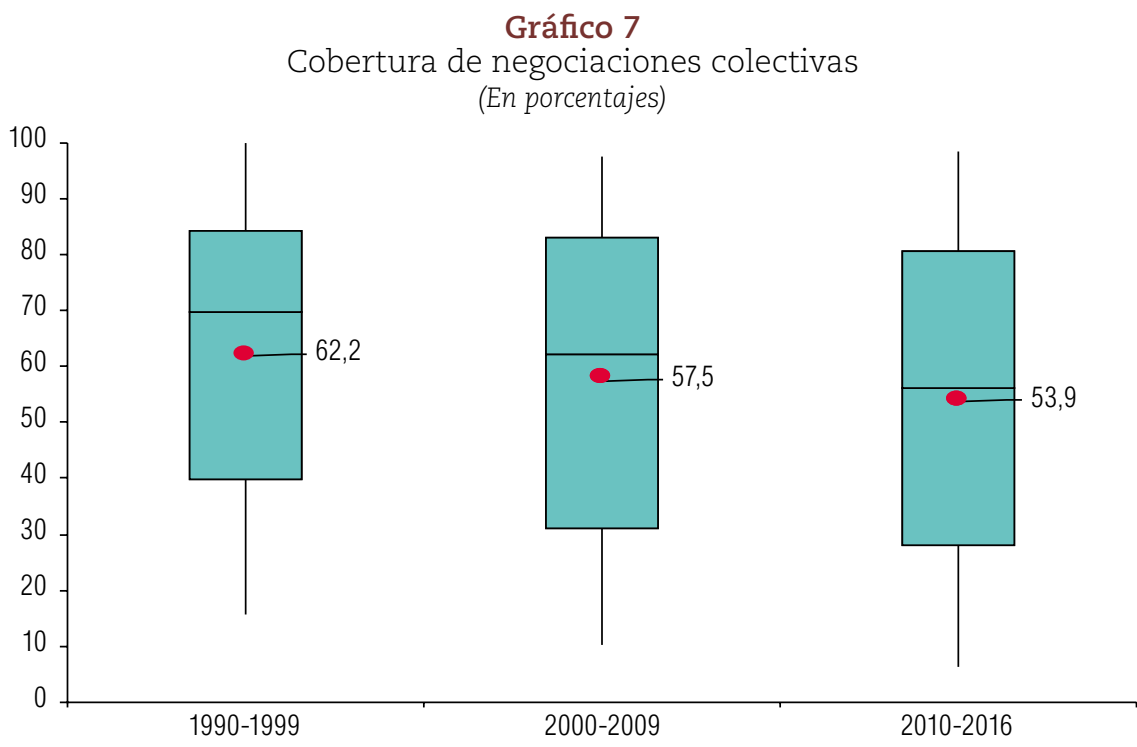

Fuente: Elaboración propia, sobre la base de información de la Organización de Cooperación y Desarrollo Económicos (OCDE). Nota: Los rectángulos y el indicador central denotan el rango del intercuartil y la mediana de la distribución, respectivamente. Los marcadores corresponden a la media por período. La muestra de países incluye: Alemania, Australia, Canadá, Chequia, Dinamarca, Eslovenia, España, Estados Unidos, Finlandia, Francia, Grecia, Hungría, Islandia, Japón, Luxemburgo, Noruega, Nueva Zelandia, Países Bajos, Portugal, Reino Unido, República de Corea, Suecia, Suiza y Turquía.

El debilitamiento del poder de negociación de los trabajadores también puede asociarse al proceso de financierización que tuvo lugar en las últimas décadas, a través de dos vías posibles (Stockhammer, 2013): i) mayores posibilidades de inversión en activos financieros y en el exterior, que plantean incentivos para una menor inversión en bienes internos reales y favorecen las opciones a corto plazo, y ii) aumento del poder relativo de los accionistas, a partir de cambios en las normas de funcionamiento internas de las empresas y del desarrollo del mercado de capitales, de manera que se asegura el pago de dividendos y se presiona sobre estrategias de reducción de costos (Lazonick y O’Sullivan, 2000).

Al tomar como variable sustitutiva de la globalización el grado de apertura comercial y de la financierización a los activos y pasivos externos sobre el producto interno bruto (PIB), en línea con 
Stockhammer (2013), se observa que ambos indicadores - asociados negativamente al poder de negociación de los trabajadores - tendieron a aumentar en los últimos años. Este incremento fue mayor en el caso de los países donde se implementaron regímenes de metas de inflación, en los que se registró una mayor disminución del traspaso del tipo de cambio (véanse los gráficos 8 y 9 ).

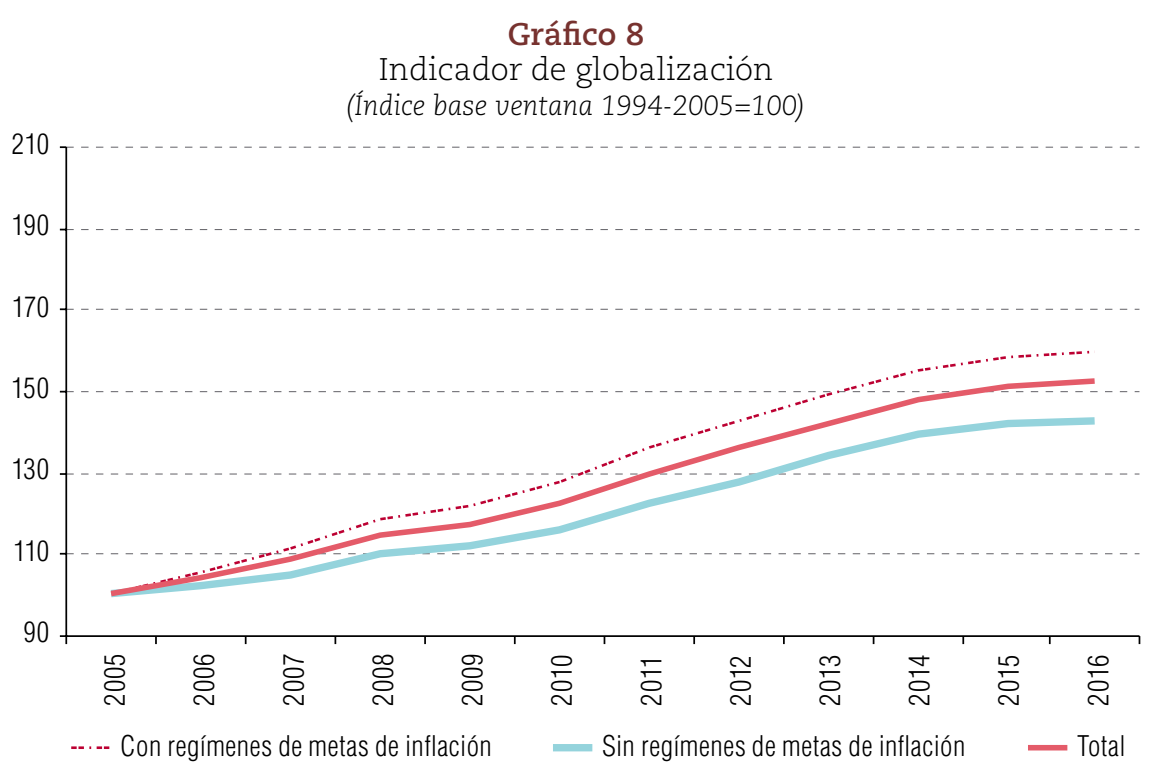

Fuente: Elaboración propia, sobre la base de datos del Fondo Monetario Internacional (FMI) y el Banco Mundial.

Nota: Las series corresponden a índices sobre el promedio móvil de 12 años a partir de 1994. El producto interno bruto (PIB) utilizado como denominador del indicador de financierización se encuentra ajustado por paridad del poder adquisitivo (PPA). En los países con regímenes de metas de inflación (implementados en 2010 o con anterioridad) se incluye a: Brasil, Chile, Colombia, Filipinas, Hungría, Indonesia, México, Perú, Polonia, Rumania, Sudáfrica, Tailandia y Turquía. En los países sin regímenes de metas de inflación (o implementados desde 2011) se incluye a: Argentina, Bolivia (Estado Plurinacional de), Bulgaria, Croacia, Federación de Rusia, India, Malasia, Paraguay y Uruguay.

\section{Gráfico 9}

Indicador de financierización

(Índice base ventana 1994-2005=100)

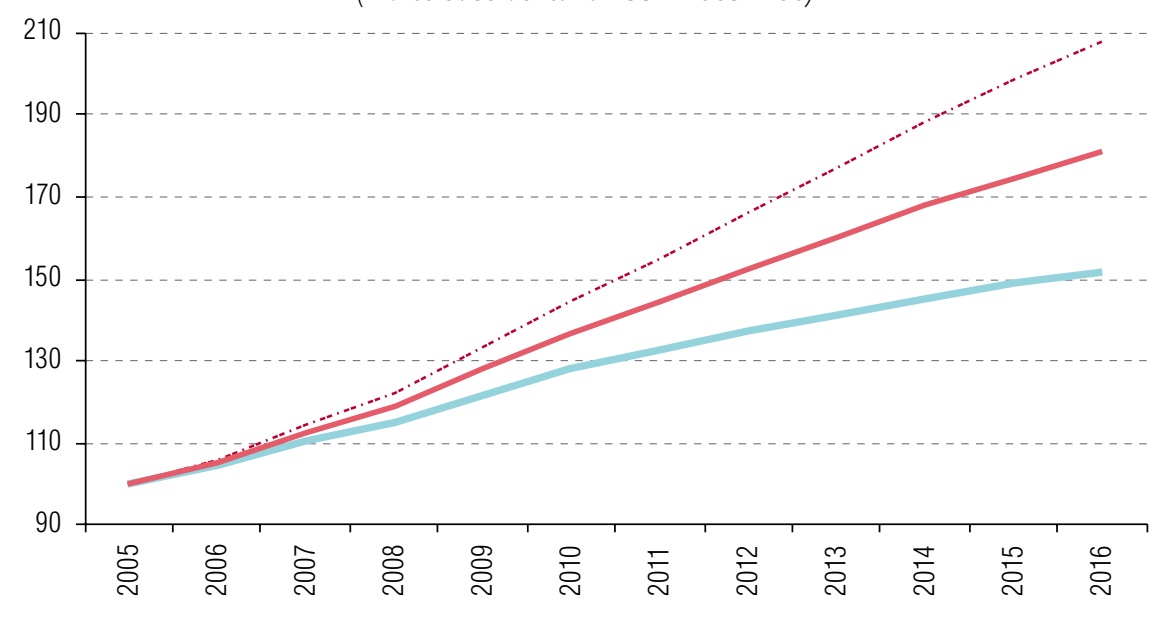

....- Con regímenes de metas de inflación — Sin regímenes de metas de inflación — Total

Fuente: Elaboración propia, sobre la base de datos del Fondo Monetario Internacional (FMI) y el Banco Mundial.

Nota: Las series corresponden a índices sobre el promedio móvil de 12 años a partir de 1994. El producto interno bruto (PIB) utilizado como denominador del indicador de financierización se encuentra ajustado por paridad del poder adquisitivo (PPA). En los países con regímenes de metas de inflación (implementados en 2010 o con anterioridad) se incluye a: Brasil, Chile, Colombia, Filipinas, Hungría, Indonesia, México, Perú, Polonia, Rumania, Sudáfrica, Tailandia y Turquía. En los países sin regímenes de metas de inflación (o implementados desde 2011) se incluye a: Argentina, Bolivia (Estado Plurinacional de), Bulgaria, Croacia, Federación de Rusia, India, Malasia, Paraguay y Uruguay. 
En particular, el indicador de apertura comercial (globalización) cobra mayor relevancia para asociar la disminución del traspaso del tipo de cambio con el debilitamiento de las instituciones laborales si se tiene en cuenta que, desde el punto de vista de las decisiones de precios, la apertura comercial debería haber incrementado la competencia entre las empresas que producen localmente y en el exterior (Benigno y Faia, 2016).

En un proceso inflacionario, el poder de negociación de los trabajadores se refleja en su capacidad para moderar o neutralizar su pérdida de ingresos reales a lo largo del tiempo. Este aspecto de la puja distributiva resulta fundamental en la determinación de la intensidad del efecto de segunda ronda originado en un incremento del tipo de cambio (véase la sección II).

Con el objetivo de evaluar la evolución de la resistencia salarial en los últimos años se estimó la expresión (4.1) a partir del método de proyección local y de series trimestrales de salarios nominales utilizando ventanas móviles (véase el anexo A2) para un subconjunto de diez países sobre los cuales se cuenta con datos suficientes 2324 .

$$
\ln w_{i, t+h-1}-\ln w_{i, t-1}=\alpha_{0}+\sum_{j=1}^{j}\left(\alpha_{1, j}^{h} p_{i, t-j}\right)+\alpha_{2}^{h} p r_{i, t}+\alpha_{3}^{h} u_{i, t}+v_{i, t}
$$

Donde $w$ es el salario nominal, $p$ es la diferencia logarítmica del índice de precios al consumidor, $p r$ es la diferencia logarítmica de la productividad laboral, $u$ es la tasa de desempleo, $v$ es un término de error, $h$ es el horizonte de proyección, $i$ cada uno de los países incluidos en el panel y $j$ la cantidad de rezagos. La cantidad de rezagos considerada para el índice de precios al consumidor es de dos $(j=2)$ y el horizonte de proyección elegido es de cuatro trimestres $(h=4)$. Nos interesa $\alpha_{1, j=1}^{h=4}$, un parámetro que refleja la magnitud de la respuesta del salario nominal acumulada a un año después de un incremento en los precios al consumidor; utilizamos este parámetro como variable sustitutiva de la resistencia salarial.

Las tasas de desempleo y los datos sobre productividad laboral se calcularon como series anuales mediante el filtro de Hodrick-Prescott (la insuficiencia de datos trimestrales impidió una mayor frecuencia). Las series de salarios nominales se obtuvieron de organismos internacionales y dependencias oficiales nacionales (véase el anexo A2).

Los resultados obtenidos muestran una reducción de la resistencia salarial en los últimos años, en línea con la evidencia sobre el debilitamiento de las instituciones laborales y con la disminución del traspaso del tipo de cambio para ese mismo conjunto de países (véase el gráfico 10). Este resultado sugiere que la menor resistencia salarial a la inflación pasada puede haber sido un factor relevante en la disminución del traspaso del tipo de cambio.

La disminución de la resistencia salarial fue más marcada en los países donde se implementaron regímenes de metas de inflación (véase el gráfico 11). Este resultado sugiere que la mayor disminución del traspaso del tipo de cambio en los países donde se adoptaron estos regímenes podría asociarse con la menor resistencia salarial, vinculada a los cambios en las instituciones laborales. Asimismo, este resultado se encuentra en línea con el mayor incremento de los indicadores de globalización y financierización - asociados negativamente con el poder de negociación de los trabajadores - en el grupo de países donde se implementaron regímenes de metas de inflación. En la siguiente sección se contrasta empíricamente esta hipótesis.

\footnotetext{
${ }^{23}$ Países con series de salarios nominales trimestrales que abarquen un período de 20 años o mayor.

${ }^{24}$ Como antecedente se puede mencionar el trabajo de Vogel y otros (2009), que estiman la resistencia del salario real a cambios en los términos de intercambio en los países del Grupo de los Siete (G7) para analizar los efectos inflacionarios de segunda ronda generados por la suba de los precios de los productos básicos en la primera mitad de 2008. La estimación se realiza a partir de una ecuación de rezagos distribuidos autorregresiva y revela que el parámetro asociado a la resistencia del salario real tendió a disminuir desde la década de 1970.
} 


\section{Gráfico 10}

Traspaso del tipo de cambio, resistencia salarial y contenido importado del consumo privado

(En porcentajes)

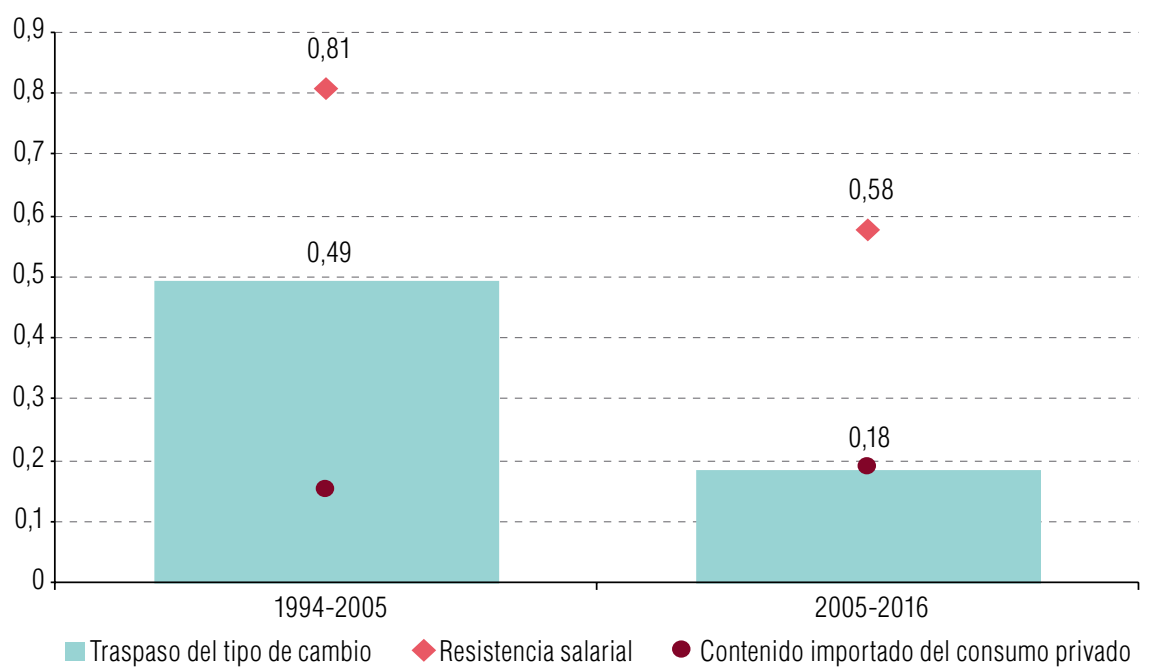

Fuente: Elaboración propia.

Nota: El traspaso del tipo de cambio refleja la respuesta acumulada de los precios al consumidor un año después de un aumento del $1 \%$ del tipo de cambio nominal efectivo. El contenido importado refleja la participación de las importaciones (intermedias y finales) en el consumo privado. La resistencia salarial refleja la respuesta acumulada de los salarios nominales un año después de un aumento del 1\% de los precios al consumidor. En las estimaciones del traspaso del tipo de cambio y de la resistencia salarial se incluyeron efectos fijos por país. Las estimaciones incluyen a: Argentina, Brasil, Chile, Colombia, Croacia, Hungría, Polonia, Rumania, Federación de Rusia y Uruguay.

\section{Gráfico 11}

Traspaso del tipo de cambio, resistencia salarial y componente importado por régimen monetario

\section{(En porcentajes)}

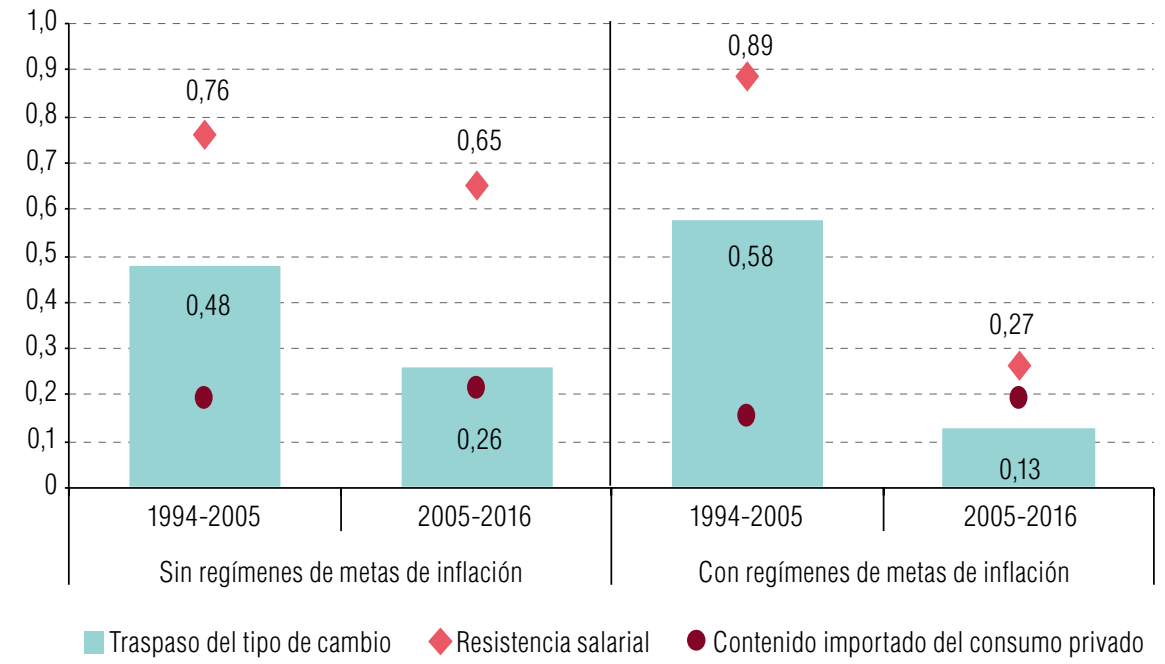

Fuente: Elaboración propia.

Nota: El traspaso del tipo de cambio refleja la respuesta acumulada de los precios al consumidor un año después de un aumento del $1 \%$ del tipo de cambio nominal efectivo. El contenido importado refleja la participación de las importaciones (intermedias y finales) en el consumo privado. La resistencia salarial refleja la respuesta acumulada de los salarios nominales un año después de un aumento del 1\% de los precios al consumidor. En los países sin regímenes de metas de inflación se incluye a: Argentina, Croacia, Federación de Rusia y Uruguay. En países con regímenes de metas de inflación se incluyen a: Brasil, Chile, Colombia, Hungría, Polonia y Rumania. En las estimaciones del traspaso del tipo de cambio y de la resistencia salarial se incluyeron efectos fijos por país. 


\section{Vínculo entre la resistencia salarial y el traspaso del tipo de cambio}

Para evaluar los factores determinantes del traspaso del tipo de cambio se sigue una estrategia similar a la aplicada por Carrière-Swallow y otros (2016) y Choudhri y Hakura (2006), que consiste en dos pasos. En primer lugar, se estiman el traspaso del tipo de cambio y la resistencia salarial para cada país en ventanas móviles de 12 años a partir de las ecuaciones (3.1) y (4.1), respectivamente. Las ventanas móviles comienzan en el primer trimestre de cada año desde 1994. Esto significa que son 12 ventanas temporales: la primera comprende el período 1994-2005, la segunda el período 1995-2006 y la última el período 2005-2016.

En segundo lugar, se estima una regresión del conjunto de coeficientes de traspaso del tipo de cambio sobre el conjunto de coeficientes de resistencia salarial obtenidos en la primera etapa (5.1). También se consideran como variables explicativas el indicador de globalización (presentado en la sección IV) y otro conjunto de variables establecidas como determinantes del traspaso del tipo de cambio en la literatura, como la media y la desviación estándar de la inflación (volatilidad de la tasa de inflación) y la media y la desviación estándar de la variación del tipo de cambio (volatilidad del tipo de cambio) ${ }^{25}$. Estas últimas también se calculan para las mismas ventanas temporales consideradas para el traspaso del tipo de cambio y la resistencia salarial. Las estimaciones se realizan para un subconjunto de diez países respecto a los cuales se dispone de datos sobre salarios nominales trimestrales (véase el anexo A2).

$$
\beta_{1, i, \tau}^{h=4}=\delta \chi_{i, \tau}+\varsigma_{\tau}+\epsilon_{i, \tau}
$$

Donde $\beta_{1, i, \tau}^{h=4}$ son los coeficientes de traspaso del tipo de cambio, y $X_{i \tau}$ y $\delta$ son cada variable explicativa (media y desviación estándar de la inflación y de la tasa de variación del tipo de cambio nominal, indicador de globalización y resistencia salarial) y su respectivo coeficiente. Las observaciones corresponden a cada ventana temporal $(\tau)$ y a cada país de la muestra (i). La especificación incluye efectos fijos temporales $\left(\varsigma_{\tau}\right)$.

Los resultados de las estimaciones de la ecuación 5.1 muestran que cada una de las variables consideradas resulta significativa cuando se introduce individualmente (véanse las columnas 1-6 del cuadro 3). Cuando se introducen todas las variables explicativas juntas y se controla por efectos fijos temporales, resultan significativas la depreciación promedio y el indicador de resistencia salarial (véase la columna 7 del cuadro 3). Cuando además se incluyen efectos fijos por país para controlar por características estructurales de cada uno de ellos, resultan significativas la volatilidad del tipo de cambio, la inflación promedio, el indicador de globalización y la resistencia salarial (véase la columna 8 del cuadro 3). En el anexo A3 se incluyen, de forma complementaria, estimaciones que consideran el tipo de cambio nominal bilateral en lugar del tipo de cambio nominal efectivo, en las que se obtienen resultados en esta misma línea.

Los coeficientes de depreciación promedio, volatilidad del tipo de cambio y nivel promedio de la inflación, correspondientes a aquellas estimaciones en las que estas variables resultan significativas, tienen signo positivo de acuerdo con lo esperado y en línea con la literatura empírica: a mayor inestabilidad cambiaria y a mayor nivel de inflación de partida, más intenso es el traspaso de la devaluación sobre los precios internos al consumidor.

\footnotetext{
${ }^{25}$ En la literatura se sostiene que, a mayor inflación y variación del tipo de cambio, y a mayor volatilidad de ambos, las empresas tienden a ajustar sus precios más frecuentemente y el traspaso del tipo de cambio sobre los precios internos tiende a ser mayor.
} 
Cuadro 3

Determinantes del traspaso total del tipo de cambio nominal efectivo

\begin{tabular}{|c|c|c|c|c|c|c|c|c|}
\hline $\begin{array}{l}\text { Variable dependiente: } \\
\beta_{1, i, \tau}^{h=4}\end{array}$ & 1 & 2 & 3 & 4 & 5 & 6 & 7 & 8 \\
\hline \multirow[t]{2}{*}{ Depreciación promedio } & $11,423^{\star \star \star}$ & & & & & & $11,594^{\star \star *}$ & 1,344 \\
\hline & $(1,58)$ & & & & & & $(2,93)$ & $(3,15)$ \\
\hline \multirow{2}{*}{$\begin{array}{l}\text { Volatilidad del tipo de } \\
\text { cambio nominal efectivo }\end{array}$} & & $2,384^{\star \star \star}$ & & & & & $-0,435$ & $2,235^{\star *}$ \\
\hline & & $(0,75)$ & & & & & $(0,74)$ & $(1,02)$ \\
\hline \multirow[t]{2}{*}{ Inflación promedio } & & & $0,710^{\star \star \star}$ & & & & 2,468 & $7,636^{\star \star \star}$ \\
\hline & & & $(1,16)$ & & & & $(2,37)$ & $(2,65)$ \\
\hline \multirow[t]{2}{*}{ Volatilidad de la inflación } & & & & $4,752^{* * *}$ & & & $-0,949$ & $-1,466$ \\
\hline & & & & $(0,92)$ & & & $(1,33)$ & $(1,38)$ \\
\hline \multirow{2}{*}{$\begin{array}{l}\text { Indicador de } \\
\text { globalización }\end{array}$} & & & & & $1,051^{\star \star *}$ & & 0,132 & $-0,967^{\star \star \star}$ \\
\hline & & & & & $(0,37)$ & & $(0,10)$ & $(0,30)$ \\
\hline \multirow[t]{2}{*}{ Resistencia salarial } & & & & & & $0,072^{\star \star \star}$ & $0,079^{\star *}$ & $0,100^{\star \star \star}$ \\
\hline & & & & & & $(0,03)$ & $(0,04)$ & $(0,04)$ \\
\hline Efectos fijos por país & No & No & No & No & No & No & No & Sí \\
\hline Efectos fijos temporales & Sí & Sí & Sí & Sí & Sí & Sí & Sí & Sí \\
\hline Observaciones & 118 & 118 & 118 & 118 & 118 & 118 & 118 & 118 \\
\hline R-cuadrado & 0,861 & 0,805 & 0,886 & 0,832 & 0,802 & 0,808 & 0,820 & 0,910 \\
\hline
\end{tabular}

Fuente: Elaboración propia.

Nota: La variable dependiente es el coeficiente de traspaso total del tipo de cambio a los precios al consumidor para la ventana de tiempo $\tau$ y el país $i$. Error estándar entre paréntesis. Significación al $1 \%\left(^{\star * *}\right)$, el $5 \%\left(^{* \star}\right)$ y el $10 \%\left(^{*}\right)$. Se excluyeron las observaciones extremas en que el coeficiente de traspaso del tipo de cambio a los precios al consumidor era superior a 1.

La resistencia salarial resulta significativa en las dos estimaciones que incluyen todas las variables (con y sin efectos fijos por país) y el signo del coeficiente obtenido es positivo de acuerdo con lo planteado en nuestra hipótesis: cuanto menor es la capacidad (poder de negociación) de recomposición salarial tras una devaluación, menos intenso es el traspaso del tipo de cambio de segunda ronda. Por otra parte, el indicador de globalización resulta significativo en la estimación que incluye efectos fijos por país y su coeficiente tiene signo negativo. Este resultado puede interpretarse de la siguiente forma: a mayor apertura comercial, mayor es la competencia indirecta entre trabajadores a nivel mundial, mayor es la presión para la flexibilización del mercado laboral local, menor es el poder de negociación salarial y menos intenso es el traspaso del tipo de cambio de segunda ronda.

En términos generales, estos resultados sugieren que, dados el nivel y la volatilidad de la inflación y del tipo de cambio, a menor resistencia salarial (y a mayor apertura comercial), menos intenso es el traspaso del tipo de cambio sobre los precios internos al consumidor. El debilitamiento de las instituciones laborales - asociado a estas dos variables - es un fenómeno de alcance mundial que puede haber contribuido de manera significativa a explicar el menor traspaso del tipo de cambio a los precios internos observado en los últimos años. 


\section{Reflexiones finales}

En este trabajo se presenta una interpretación alternativa de la disminución de la intensidad del traspaso (pass-through) del tipo de cambio nominal a los precios al consumidor observada en las últimas décadas en los países periféricos. Según el enfoque convencional, esa disminución se debió a la mayor credibilidad de las autoridades monetarias, que en la literatura se suele asociar al establecimiento de regímenes formales de metas de inflación y a las reformas institucionales vinculadas (entre ellas, independencia del banco central, libre movilidad internacional de capitales y flotación cambiaria). Desde una perspectiva afín a la tradición estructuralista - en cuyo análisis de la inflación se atribuye un papel fundamental al conflicto distributivo-, en este trabajo se dirigió la atención a otras transformaciones estructurales, que se registraron en paralelo a la instauración de los regímenes de metas de inflación y se tradujeron en una menor resistencia salarial, una variable clave en la explicación de la intensidad del traspaso cambiario. Entre esas transformaciones se destaca el debilitamiento de las instituciones laborales, incluida la reducción de la densidad sindical.

La evidencia empírica obtenida muestra que la disminución de la intensidad del traspaso del tipo de cambio se produjo tanto en los países que instauraron regímenes formales de metas de inflación como en los que no lo hicieron. Eso indicaría que dicha disminución se debió a un proceso más amplio, de alcance mundial, no necesariamente derivado de una mayor credibilidad de las autoridades monetarias, que podría incluir una apertura comercial generalizada, una creciente desregulación financiera o el aumento de la fuerza de trabajo mundial. La información recabada revela, además, que la disminución del traspaso cambiario fue más significativa en los países donde se instauraron regímenes formales de metas de inflación que en el resto de los países de la muestra.

En línea con la hipótesis convencional, ello podría reflejar que, sin perjuicio de la posible incidencia de los factores globales mencionados, la instauración de regímenes de metas de inflación puede haber contribuido a reducir el traspaso del tipo de cambio con mayor intensidad. Sin embargo, un examen más detallado de la evidencia empírica presentada en las secciones IV y $\vee$ sugiere que: i) la disminución de la resistencia salarial se produjo con mayor intensidad precisamente en los países donde se implementaron regímenes formales de metas de inflación, y ii) la resistencia salarial es un factor significativo desde el punto de vista estadístico para explicar la intensidad del traspaso del tipo de cambio. De ello podría inferirse que la reducción del traspaso del tipo de cambio a los precios se manifiesta con mayor intensidad en ese subgrupo de países debido a que en estos fue más significativa la reducción de la capacidad de resistencia salarial $-\mathrm{y}$, presumiblemente, más acentuadas las transformaciones estructurales e institucionales que ocasionaron dicha reduccióny no a la mayor credibilidad de sus instituciones monetarias.

Aunque probablemente no constituyan una sorpresa, estos resultados pueden ser de interés para quienes analizan de manera comparada las distintas formas institucionales capitalistas, como, por ejemplo, los partidarios de la teoría francesa de la regulación o del llamado enfoque de "variedades de capitalismo"26. En este último, por ejemplo, se hace hincapié en la noción de complementariedades institucionales, que remite a la manera en que el funcionamiento y el desempeño de una institución pueden incidir en el funcionamiento y el desempeño de otra. El análisis macroeconómico convencional parece por momentos asumir una perspectiva similar, al sugerir un conjunto de condiciones que una economía debe satisfacer para que la instrumentación de un régimen formal de metas de inflación redunde en una tasa inflacionaria baja y estable. Entre estas condiciones suelen destacarse la independencia de la autoridad monetaria, la liberalización de la cuenta de capital de la balanza de pagos, la libre flotación del tipo de cambio y la autoimposición de límites en materia fiscal27.

\footnotetext{
${ }^{26}$ Véase una aplicación del enfoque de variedades de capitalismo al caso de los países de América Latina en Schneider (2009). Véase una reseña crítica de este abordaje en relación con su aplicabilidad en la región en Aguirre y Lo Vuolo (2013). Véase un enfoque alternativo sobre las economías de la región, basado en la teoría de la regulación, en Bizberg y Théret (2012).

27 Véase, por ejemplo, Fraga, Goldfajn y Minella (2003).
} 
De la evidencia analizada en este trabajo se deduce otra precondición institucional, de igual o mayor relevancia que las mencionadas: la desregulación del mercado laboral28. Esta precondición no suele aparecer de manera explícita en la literatura convencional, ni es preconizada abiertamente por los partidarios de los regímenes de metas de inflación, aun cuando resultaría perfectamente coherente con la explicación convencional. En efecto, una mayor credibilidad de la autoridad monetaria puede interpretarse en el sentido de que las aspiraciones salariales de los trabajadores se han alineado con la meta oficial de inflación, ya sea de manera espontánea o por medio de alguna forma de representación sindical. Ello implica, por ejemplo, y para retomar la discusión sobre las causas de la disminución del traspaso del tipo de cambio a los precios internos, que frente a una depreciación cambiaria los trabajadores ofrezcan la menor resistencia salarial posible, aceptando de manera apacible cierta disminución de sus ingresos reales ante la certeza de que la alternativa a esa pasividad sería una política monetaria contractiva que eventualmente alcanzará el mismo resultado (en términos salariales y de la tasa de inflación) pero con un costo mucho mayor en términos de empleo y actividad ${ }^{29}$. En suma, la disminución del traspaso del tipo de cambio, que desde la perspectiva convencional se presenta como un atributo de la credibilidad monetaria, es en la práctica un efecto del debilitamiento de las instituciones laborales.

\section{Bibliografía}

Aguirre, J. y R. Lo Vuolo (2013), "Variedades de capitalismo: una aproximación al estudio comparado del capitalismo y sus aplicaciones para América Latina", Documentos de Trabajo CIEPP, № 85, Buenos Aires, Centro Interdisciplinario para el Estudio de Políticas Públicas (CIEPP).

Arestis, P. y M. Sawyer (2005), "Aggregate demand, conflict and capacity in the inflationary process", Cambridge Journal of Economics, vol. 29, № 6, Oxford, Oxford University Press.

Bailliu, J. y E. Fujii (2004), "Exchange rate pass-through and the inflation environment in industrialized countries: an empirical investigation", Bank of Canada Working Paper, № 2004-21, Ottawa, Banco de Canadá.

Bastian, E. y M. Setterfield (2017), "Nominal exchange rate shocks and inflation in an open economy: towards a structuralist inflation targeting agenda", NSSR Working Paper, № 20/2017, Nueva York, The New School for Social Research.

Benigno, P. y E. Faia (2016), "Globalization, pass-through, and inflation dynamics", International Journal of Central Banking, vol. 12, № 4.

Berg, J. (ed.) (2015), Labour Markets, Institutions and Inequality: Building Just Societies in the 21st Century, Cheltenham, Edward Elgar/Organización Internacional del Trabajo (OIT).

Bhaduri, A. y S. Marglin (1990), "Unemployment and the real wage: the economic basis for contesting political ideologies", Cambridge Journal of Economics, vol. 14, № 4, Oxford, Oxford University Press.

Bizberg, I. y B. Théret (2012), "La diversité des capitalismes latino-américains : les cas de l'Argentine, du Brésil et du Mexique", Revue de la Régulation, № 11, París, Maison des Sciences de l'Homme Paris Nord.

Blecker, R. (2011), "Open economy models of distribution and growth", A Modern Guide to Keynesian Macroeconomics and Economic Policies, E. Hein y E. Stockhammer (eds.), Cheltenham, Edward Elgar. (1989), "International competition, income distribution and economic growth", Cambridge Journal of Economics, vol. 13, № 3, Oxford, Oxford University Press.

Borensztein, E. y V. Queijo von Heideken (2016), "Exchange rate pass-through in South America: an overview", IDB Working Paper, № 710, Washington, D.C., Banco Interamericano de Desarrollo (BID).

Burstein, A., M. Eichenbaum y S. Rebelo (2005), "Large devaluations and the real exchange rate", Journal of Political Economy, vol. 113, N 4, Chicago, University of Chicago Press.

Calvo, G. y C. Reinhart (2002), "Fear of floating", The Quarterly Journal of Economics, vol. 117, № 2, Oxford, Oxford University Press.

Campa, J. y L. Goldberg (2005), "Exchange rate pass-through into import prices", Review of Economics and Statistics, vol. 87, № 4, Cambridge, Massachusetts, MIT Press.

\footnotetext{
${ }^{28}$ Véase un razonamiento similar en Hall y Gingerich (2009).

${ }^{29}$ Lo que en la literatura se conoce como ratio de sacrificio (sacrifice ratio).
} 
Carlin, W. y D. Soskice (2006), Macroeconomics: Imperfections, Institutions, and Policies, Oxford, Oxford University Press.

Carrière-Swallow y otros (2016), "Monetary policy credibility and exchange rate pass-through", IMF Working Paper, $N^{\circ} 16 / 240$, Washington, D.C., Fondo Monetario Internacional (FMI).

Caselli, F. y A. Roitman (2016), "Non-linear exchange rate pass-through in emerging markets", IMF Working Paper, No 16/1, Washington, D.C., Fondo Monetario Internacional (FMI).

Cassetti, M. (2003), "Bargaining power, effective demand and technical progress: a Kaleckian model of growth", Cambridge Journal of Economics, vol. 27, № 3, Oxford, Oxford University Press.

Choudhri, E. y D. Hakura (2006), "Exchange rate pass-through to domestic prices: does the inflationary environment matter?", Journal of international Money and Finance, vol. 25, № 4, Amsterdam, Elsevier. (2001), "Exchange rate pass-through to domestic prices: does the inflationary environment matter?", IMF Working Paper, N ${ }^{\circ}$ 01/194, Washington, D.C., Fondo Monetario Internacional (FMI).

Cornwall, J. (1990), The Theory of Economic Breakdown, Oxford, Basil Blackwell.

Cornwall, J. y W. Cornwall (2001), Capitalist Development in the Twentieth Century, Cambridge, Cambridge University Press.

Devereux, M. y J. Yetman (2010), "Price adjustment and exchange rate pass-through", Journal of International Money and Finance, vol. 29, № 1, Amsterdam, Elsevier.

Dornbusch, R. (1987), "Exchange rate and prices", American Economic Review, vol. 77, № 1, Nashville, Tennessee, American Economic Association.

Driscoll, J. y A. Kraay (1998), "Consistent covariance matrix estimation with spatially dependent panel data", Review of Economics and Statistics, vol. 80, № 4, Cambridge, Massachusetts, MIT Press.

Dutt, A. (1987), "Alternative closures again: a comment on 'Growth, distribution and inflation'”, Cambridge Journal of Economics, vol. 11, № 1, Oxford, Oxford University Press.

Dvoskin, A. y G. Feldman (2015), "Política cambiaria, distribución del ingreso y estructura productiva", Estructura productiva y política macroeconómica: enfoques heterodoxos desde América Latina, A. Bárcena, A. Prado y M. Abeles (eds.), Libros de la CEPAL, № 138 (LC/G.2653-P), Santiago, Comisión Económica para América Latina y el Caribe (CEPAL).

Fischer, S., R. Sahay y C. Vegh (2002), "Modern hyper-and high inflations", NBER Working Paper, № 8930, Cambridge, Massachusetts, Oficina Nacional de Investigaciones Económicas (NBER).

Fraga, A., I. Goldfajn y A. Minella (2003), "Inflation targeting in emerging market economies", NBER Working Paper, N N 10019, Cambridge, Massachusetts, Oficina Nacional de Investigaciones Económicas (NBER).

Frankel, J., D. Parsley y S. Wei (2012), "Slow pass-through around the world: a new import for developing countries?", Open Economies Review, vol. 23, № 2, Nueva York, Springer.

Freeman, R. (2006), "The great doubling: the challenge of the new global labor market" [en línea] https:// www.researchgate.net/publication/237491969.

Frenkel, R. (1984), "Inflación, shocks y mark-up: Argentina 1975-1982”, Ensayos Económicos, №30, Buenos Aires, Banco Central de la República Argentina.

Furtado, C. (1963), The Economic Growth of Brazil, Berkeley, University of California Press.

Gagnon, J. y J. Ihrig (2004), "Monetary policy and exchange rate pass-through", International Journal of Finance \& Economics, vol. 9, № 4, Hoboken, Wiley.

Godley, W. y M. Lavoie (2007), Monetary Economics: An Integrated Approach to Credit, Money, Income, Production and Wealth, Nueva York, Palgrave Macmillan.

Gopinath, G. (2015), "The international price system", NBER Working Paper, № 21646, Cambridge, Massachusetts, Oficina Nacional de Investigaciones Económicas (NBER).

Hall, P. y D. Gingerich (2009), "Varieties of capitalism and institutional complementarities in the political economy: an empirical analysis", British Journal of Political Science, vol. 39, № 3, Cambridge, Cambridge University Press.

Jordà, Ò. (2005), "Estimation and inference of impulse responses by local projections", American Economic Review, vol. 95, № 1, Nashville, Tennessee, American Economic Association.

Kalecki, M. (1971), "Costs and prices", Selected Essays on the Dynamics of the Capitalist Economy 1933-1970, Cambridge, Cambridge University Press.

Krugman, P. (1987), "Pricing to market when the exchange rate changes", Real Financial Linkages Among Open Economies, S. Arndt y J. Richardson (eds.), Cambridge, Massachusetts, MIT Press.

Lavoie, M. (2014), Post-Keynesian Economics: New Foundations, Cheltenham, Edward Elgar. 
Lazonick, W. y M. O'Sullivan (2000), "Maximizing shareholder value: a new ideology for corporate governance", Economy and Society, vol. 29, № 1, Abingdon, Taylor \& Francis.

Lenzen, M. y otros (2013), "Building EORA: a global multi-region input-output database at high country and sector resolution", Economic Systems Research, vol. 25, № 1, Abingdon, Taylor \& Francis.

(2012), "Mapping the structure of the world economy", Environmental Science \& Technology, vol. 46, $N^{\circ} 15$, Washington, D.C., American Chemical Society.

Libman, E. (2018), "Política monetaria y cambiaria asimétrica en países latinoamericanos que usan metas de inflación", Revista CEPAL, № 125 (LC/PUB.2018/6-P), Santiago, Comisión Económica para América Latina y el Caribe (CEPAL).

Marston, R. (1990), "Pricing to market in Japanese manufacturing", Journal of International Economics, vol. 29, № 3-4, Amsterdam, Elsevier.

Naciones Unidas (2016), Informe del Experto Independiente sobre las consecuencias de la deuda externa y las obligaciones financieras internacionales conexas de los Estados para el pleno goce de todos los derechos humanos, sobre todo los derechos económicos, sociales y culturales (A/HRC/34/57), Nueva York, 27 de diciembre.

Neville, J. y P. Kriesler (2008), "Expectations and unemployment”, Keynes and Macroeconomics after 70 Years: Critical Assessments of The General Theory, L. Wray y M. Forstater (eds.), Cheltenham, Edward Elgar.

Noyola, J. (1956), "El desarrollo económico y la inflación en México y otros países latinoamericanos", Investigación Económica, vol. 16, № 4, Ciudad de México, Universidad Nacional Autónoma de México (UNAM).

Olivera, J. (1967), "Aspectos dinámicos de la inflación estructural", Desarrollo Económico, vol. 7, № 27, Buenos Aires, Instituto de Desarrollo Económico y Social (IDES).

Perry, N. y N. Cline (2016), "What caused the great inflation moderation in the US? A post-Keynesian view", Review of Keynesian Economics, vol. 4, № 4, Cheltenham, Edward Elgar.

Rochon, L. y M. Setterfield (2007), "Interest rates, income distribution, and monetary policy dominance: post Keynesians and the 'fair rate' of interest", Journal of Post Keynesian Economics, vol. 30, № 1, Abingdon, Taylor \& Francis.

Rowthorn, R. (1977), "Conflict, inflation and money", Cambridge Journal of Economics, vol. 1, № 3, Oxford, Oxford University Press.

Sarantis, N. (1990), "Distribution and terms of trade dynamics, inflation, and growth", Journal of Post Keynesian Economics, vol. 13, № 2, Abingdon, Taylor \& Francis.

Sawyer, M. (1982), Macro-Economics in Question: The Keynesian-Monetarist Orthodoxies and the Kaleckian Alternative, Nueva York, M. E. Sharpe.

Schneider, B. (2009), "Hierarchical market economies and varieties of capitalism in Latin America", Journal of Latin American Studies, vol. 41, № 3, Cambridge, Cambridge University Press.

Schönerwald da Silva, C. y M. Vernengo (2008), "The decline of the exchange rate pass-through in Brazil: explaining the 'fear of floating'”, International Journal of Political Economy, vol. 37, N 4, Abingdon, Taylor \& Francis.

Setterfield, M. (2009), "Macroeconomics without the LM curve: an alternative view", Cambridge Journal of Economics, vol. 33, № 2, Oxford, Oxford University Press.

(2006a), "Balancing the macroeconomic books on the backs of the workers: a simple analytical political economy model of contemporary US capitalism", International Journal of Political Economy, vol. 35, № 3 , Abingdon, Taylor \& Francis.

(2006b), "Is inflation targeting compatible with post Keynesian economics?", Journal of Post Keynesian Economics, vol. 28, № 4, Abingdon, Taylor \& Francis.

Smithin, J. (1994), Controversies in Monetary Economics: Ideas, Issues, and Policy, Cheltenham, Edward Elgar.

Stockhammer, E. (2013), "Why have wage shares fallen? An analysis of the determinants of functional income distribution", Wage-led Growth: An Equitable Strategy for Economic Recovery, M. Lavoie y E. Stockhammer (eds.), Londres, Palgrave Macmillan/Organización Internacional del Trabajo (OIT).

Sunkel, O. (1958), "La inflación chilena: un enfoque heterodoxo", El Trimestre Económico, vol. 25, № 4, Ciudad de México, Fondo de Cultura Económica.

Taylor, C. (1985), "Social theory as practice", Philosophy and the Human Sciences: Philosophical Papers 2, Cambridge, Cambridge University Press.

Taylor, J. (2000), "Low inflation, pass-through, and the pricing power of firms", European Economic Review, vol. 44, № 7, Amsterdam, Elsevier. 
Taylor, L. (2004), Reconstructing Macroeconomics: Structuralist Proposals and Critiques of the Mainstream, Cambridge, Massachusetts, Harvard University Press.

- (1991), Income Distribution, Inflation and Growth: Lectures on Structuralist Macroeconomic Theory, Cambridge, Massachusetts, MIT Press.

_ (1985), "A stagnationist model of economic growth", Cambridge Journal of Economics, vol. 9, № 4, Oxford, Oxford University Press.

Teulings, C. y N. Zubanov (2014), "Is economic recovery a myth? Robust estimation of impulse responses", Journal of Applied Econometrics, vol. 29, №3, Hoboken, Wiley.

Vera, L. (2014), "The simple post-Keynesian monetary policy model: an open economy approach", Review of Political Economy, vol. 26, № 4, Abingdon, Taylor \& Francis.

Vernengo, M. (2003), "Balance of payments constraint and inflation", Department of Economics Working Paper, NN2003-06, University of Utah.

Vogel, L. y otros (2009), "Inflation responses to recent shocks: do G7 countries behave differently?", OECD Economics Department Working Paper, Nº68, París, Organización de Cooperación y Desarrollo Económicos (OCDE). 


\section{Anexo A1}

\section{Obtención del salario real de equilibrio}

De acuerdo con Bastian y Setterfield (2017), se define la tasa de variación de los salarios y de los precios, solo considerando la brecha de aspiraciones en materia de ingresos:

$$
\begin{aligned}
& \hat{w}_{t}=\mu_{1}\left(\left[\omega_{w 0}+\mu_{2} e_{t-1}^{R}\right]-\omega_{t-1}\right) \\
& \hat{p}_{t}=\varphi_{1}\left(\omega_{t-1}-\left[\omega_{f 0}-\varphi_{2} e_{t-1}^{R}\right]\right)
\end{aligned}
$$

Se obtiene el salario real de equilibrio, imponiendo como condición que el salario real permanece constante $\left(\hat{w}_{t}=\hat{p}_{t}\right)$ :

$$
\omega^{*}=\frac{\mu_{1} \omega_{w 0}+\varphi_{1} \omega_{f 0}+\left(\mu_{1} \mu_{2}-\varphi_{1} \varphi_{2}\right) e_{t}^{R}}{\mu_{1}+\varphi_{1}}
$$

Se reemplaza la ecuación (A.3) en (A.2) y se obtiene la tasa de inflación de equilibrio:

$$
p^{*}=\frac{\mu_{1} \varphi_{1}\left(\omega_{w 0}-\omega_{f 0}\right)+\left(\mu_{2}+\varphi_{2}\right) e_{t}^{R}}{\mu_{1}+\varphi_{1}}
$$

Se obtiene la tasa de variación del tipo de cambio nominal de equilibrio, asumiendo que el tipo de cambio real permanece constante. Esto implica que la tasa de variación del tipo de cambio nominal es igual a la inflación, menos la tasa de variación de los precios de los productos de importación en moneda extranjera $\left(\hat{e}=\hat{p}-\hat{p}^{f}\right)$.

$$
\begin{gathered}
\hat{e}^{*}=\frac{\mu_{1} \varphi_{1}\left[\left(\omega_{w 0}-\omega_{f 0}\right)+\left(\mu_{2}+\varphi_{2}\right) e_{t}^{R}\right]}{\mu_{1}+\varphi_{1}}-\hat{p}^{f} \\
\hat{e}^{*}=\frac{\mu_{1} \varphi_{1}\left(\omega_{w 0}-\omega_{f 0}\right)+\mu_{1} \varphi_{1}\left(\mu_{2}+\varphi_{2}\right) e_{t}^{R}-\hat{p}^{f}\left(\mu_{1}+\varphi_{1}\right)}{\mu_{1}+\varphi_{1}}
\end{gathered}
$$

Luego se despeja el tipo de cambio real:

$$
e_{t}^{R *}=\left[\hat{e}^{*}-\frac{\mu_{1} \varphi_{1}\left(\omega_{w 0}-\omega_{f 0}\right)+\hat{p}^{f}\left(\mu_{1}+\varphi_{1}\right)}{\mu_{1}+\varphi_{1}}\right] \frac{\mu_{1}+\varphi_{1}}{\mu_{1} \varphi_{1}\left(\mu_{2}+\varphi_{2}\right)}
$$

Por último, se reemplaza (A.6) en (A.3):

$$
\omega^{*}=\frac{\mu_{1} \omega_{w 0}+\varphi_{1} \omega_{f 0}+\left(\mu_{1} \mu_{2}-\varphi_{1} \varphi_{2}\right)\left[\frac{\left(\hat{e}+\hat{p}_{t}^{f}\right)\left(\mu_{1}+\varphi_{1}\right)-\mu_{1} \varphi_{1}\left(\omega_{w 0}-\omega_{f 0}\right)}{\mu_{1} \varphi_{1}\left(\mu_{2}+\varphi_{2}\right)}\right]}{\mu_{1}+\varphi_{1}}
$$




\section{Anexo A2}

\section{Fuentes de datos}

Cuadro A2.1

Fuentes de datos

\begin{tabular}{llll}
\hline Variable & $\begin{array}{l}\text { Frecuencia de } \\
\text { publicación }\end{array}$ & Tratamiento & Fuente \\
\hline Índice de precios al consumidor (IPC) & Mensual & Promedio trimestral & $\begin{array}{l}\text { Fondo Monetario Internacional (FMI), } \\
\text { organismos nacionales y provinciales }\end{array}$ \\
\hline Tipo de cambio nominal efectivo (TCNE) & Mensual & Promedio trimestral & Bruegel \\
\hline Precios de productos básicos & Mensual & Promedio trimestral & FMl \\
\hline Producto interno bruto (PIB) & Trimestral & $\begin{array}{l}\text { Desestacionalización } \\
\text { (X-12-ARIMA) }\end{array}$ & $\begin{array}{l}\text { FMl, Comisión Económica para América } \\
\text { Latina y el Caribe (CEPAL), Organización } \\
\text { de Cooperación y Desarrollo Económicos } \\
\text { (OCDE) y organismos nacionales }\end{array}$ \\
\hline Desempleo & Anual & Filtro de Hodrick-Prescott & Organización Internacional del Trabajo (OIT) \\
\hline Productividad & Anual & Filtro de Hodrick-Prescott & OIT \\
\hline Flujo comercial sobre PIB & Anual & - & Banco Mundial \\
\hline Activos y pasivos externos & Anual & - & FMl \\
\hline PIB (paridad del poder adquisitivo) & Anual & - & FMl \\
\hline
\end{tabular}

Cuadro A2.2

Fuentes de las series de salarios nominales

\begin{tabular}{llll}
\hline País & Período & Descripción & Fuente \\
\hline Argentina & $1995-2016$ & Salario promedio del sector privado registrado & $\begin{array}{l}\text { Ministerio de Trabajo, Empleo y Seguridad Social } \\
\text { (MTEySS) }\end{array}$ \\
\hline Brasil & $1994-2016$ & Salario promedio privado y público & Instituto Brasileño de Geografía y Estadística (IBGE) \\
\hline Chile & $1994-2016$ & Salario promedio & Fondo Monetario Internacional (FMI) \\
\hline Colombia & $1994-2016$ & $\begin{array}{l}\text { Salario promedio (industria manufacturera y } \\
\text { comercio minorista) }\end{array}$ & Banco de la República \\
\hline Croacia & $1994-2016$ & Salario promedio neto & Oficina de Estadísticas de Croacia \\
\hline Federación de Rusia & $1994-2016$ & Salario promedio & Servicio Federal de Estadística del Estado \\
\hline Hungría & $1994-2016$ & Salario promedio & FMl \\
\hline Polonia & $1994-2016$ & Salario promedio & FMl \\
\hline Rumania & $1994-2016$ & Salario promedio & FMl \\
\hline Uruguay & $1994-2016$ & Índice medio de salarios & Ministerio de Trabajo y Seguridad Social \\
\hline
\end{tabular}




\section{Anexo A3}

\section{Control de robustez: estimación sobre la base del tipo de cambio nominal bilateral}

Los resultados basados en el tipo de cambio nominal bilateral son similares a los obtenidos con el tipo de cambio nominal efectivo, con una disminución en todos los casos y una mayor reducción en los países con regímenes de metas de inflación (véanse los gráficos A3.1 a A3.4 y el cuadro A3.1).

\section{Gráfico A3.1}

Traspaso del tipo de cambio en países periféricos en ventanas móviles

(En porcentajes)

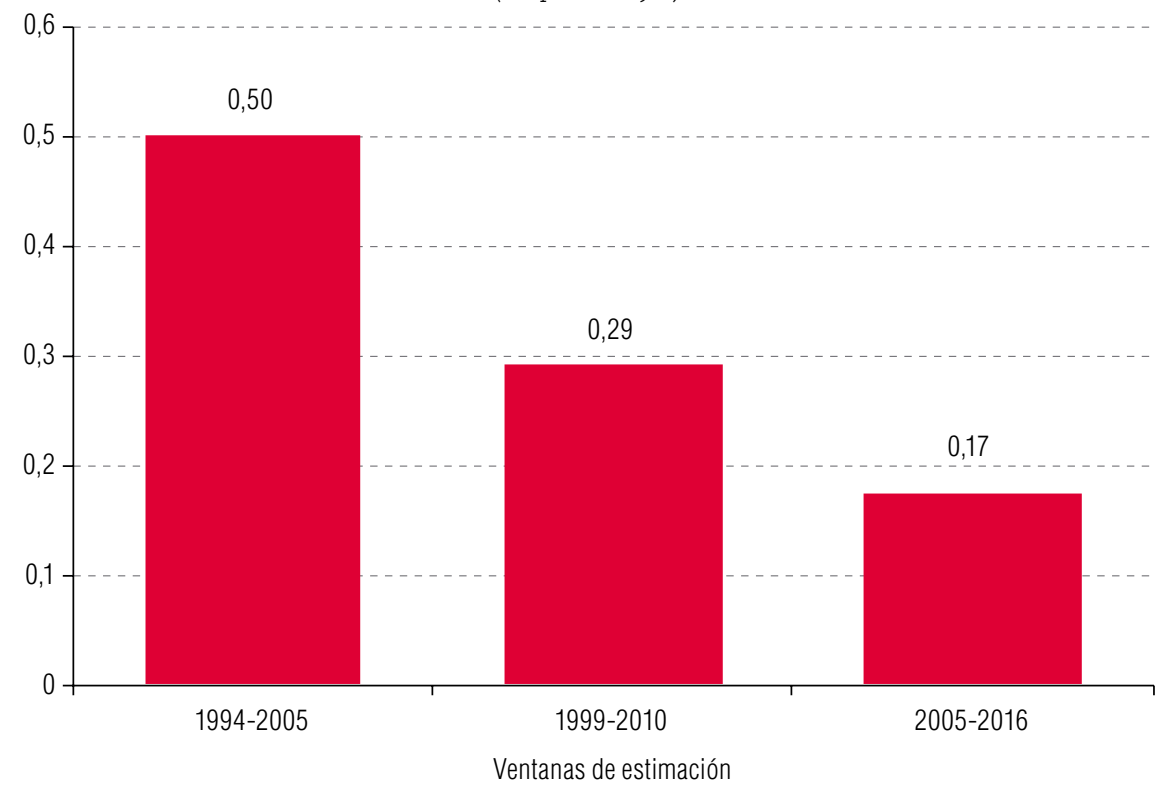

Fuente: Elaboración propia.

Nota: Respuesta acumulada de los precios al consumidor un año después de un aumento del $1 \%$ del tipo de cambio nominal bilateral. En las estimaciones se considera a los siguientes países: Argentina, Bolivia (Estado Plurinacional de), Brasil, Bulgaria, Chile, Colombia, Croacia, Federación de Rusia, Filipinas, Hungría, India, Indonesia, Malasia, México, Paraguay, Perú, Polonia, Rumania, Sudáfrica, Tailandia, Turquía y Uruguay. 


\section{Gráfico A3.2}

Traspaso del tipo de cambio en países periféricos para distintos horizontes temporales (En porcentajes)

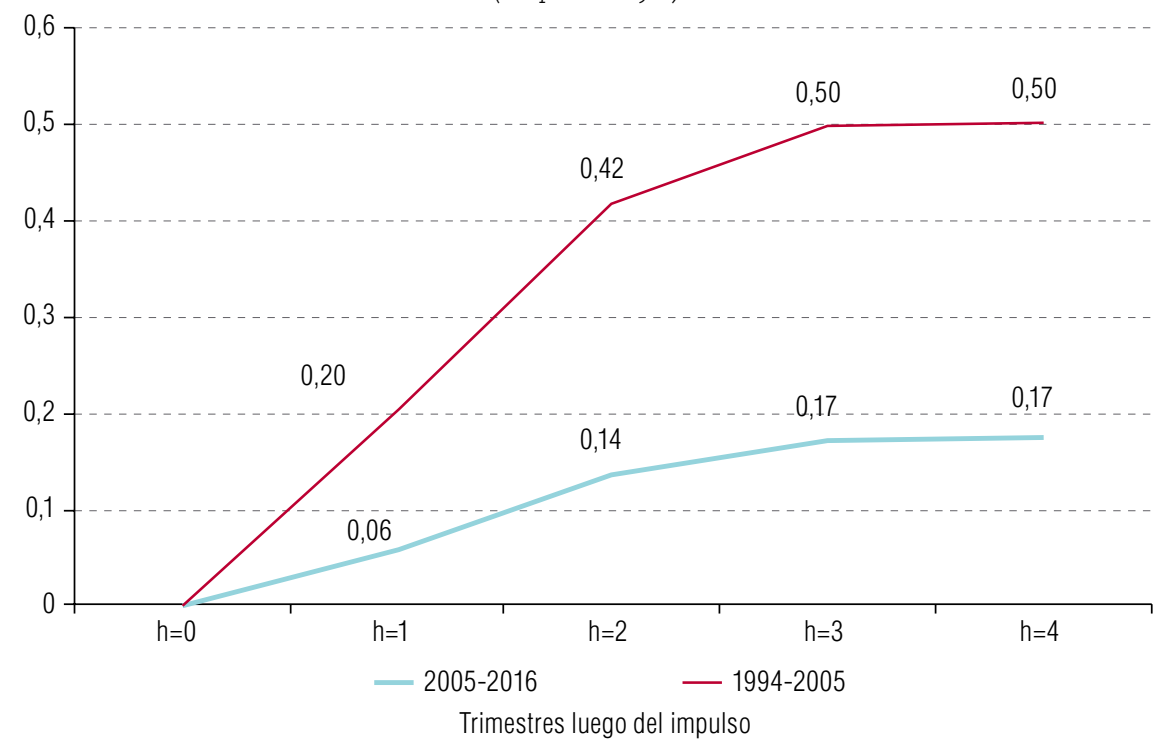

Fuente: Elaboración propia.

Nota: Respuesta acumulada de los precios al consumidor uno, dos, tres y cuatro trimestres después de un aumento del $1 \%$ del tipo de cambio nominal bilateral. En las estimaciones se considera a los siguientes países: Argentina, Bolivia (Estado Plurinacional de), Brasil, Bulgaria, Chile, Colombia, Croacia, Federación de Rusia, Filipinas, Hungría, India, Indonesia, Malasia, México, Paraguay, Perú, Polonia, Rumania, Sudáfrica, Tailandia, Turquía y Uruguay.

\section{Gráfico A3.3}

Traspaso del tipo de cambio por regiones

(En porcentajes)

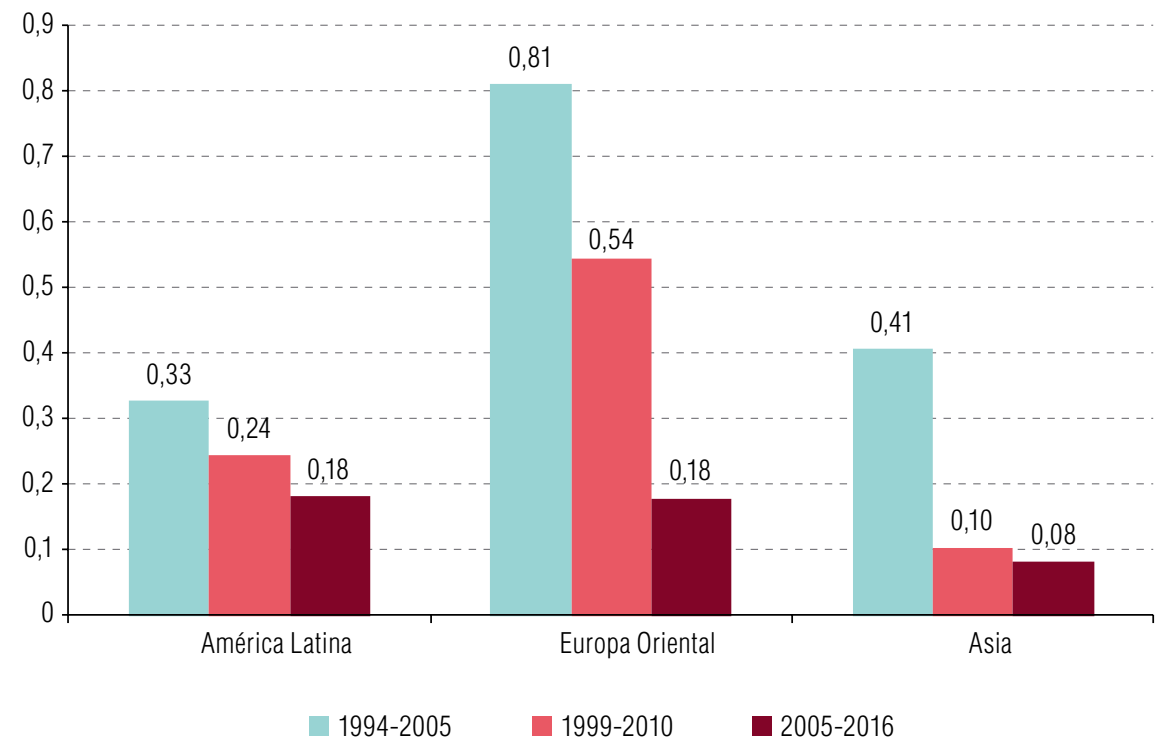

Fuente: Elaboración propia.

Nota: Respuesta acumulada de los precios al consumidor un año después de un aumento del $1 \%$ del tipo de cambio nominal bilateral. América Latina incluye: Argentina, Bolivia (Estado Plurinacional de), Brasil, Chile, Colombia, México, Paraguay, Perú y Uruguay; Europa Oriental incluye: Bulgaria, Croacia, Federación de Rusia, Hungría, Polonia, Rumania y Turquía; Asia incluye: Filipinas, India, Indonesia, Malasia y Tailandia. 
Gráfico A3.4

Traspaso del tipo de cambio por regímenes monetarios en países periféricos (En porcentajes)

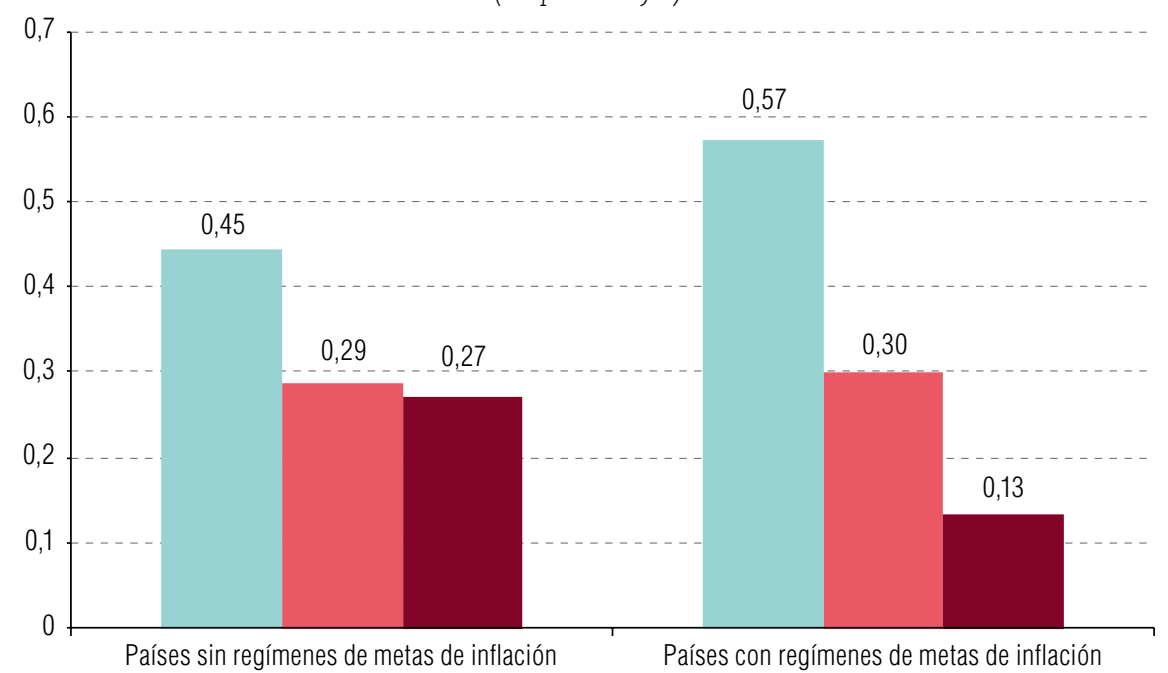

$1994-2005$

$1999-2010$

2005-2016

Fuente: Elaboración propia.

Nota: Respuesta acumulada de los precios al consumidor un año después de un aumento del $1 \%$ del tipo de cambio nominal bilateral. En los países con regímenes de metas de inflación (implementados en 2010 o con anterioridad) se incluye a: Brasil, Chile, Colombia, Filipinas, Hungría, Indonesia, México, Perú, Polonia, Rumania, Sudáfrica, Tailandia y Turquía. En los países sin regímenes de metas de inflación (o implementados desde 2011) se incluye a: Argentina, Bolivia (Estado Plurinacional de), Bulgaria, Croacia, Federación de Rusia, India, Malasia, Paraguay y Uruguay.

Cuadro A3.1

Determinantes del traspaso total del tipo de cambio nominal bilateral

\begin{tabular}{|c|c|c|c|c|c|c|c|c|}
\hline $\begin{array}{l}\text { Variable dependiente: } \\
\beta_{1, i, \tau}^{h=4}\end{array}$ & 1 & 2 & 3 & 4 & 5 & 6 & 7 & 8 \\
\hline \multirow[t]{2}{*}{ Depreciación promedio } & $11,606^{\star \star \star}$ & & & & & & $13,372^{\star \star \star}$ & 5,077 \\
\hline & $(1,74)$ & & & & & & $(3,33)$ & $(3,51)$ \\
\hline \multirow{2}{*}{$\begin{array}{l}\text { Volatilidad del tipo de cambio } \\
\text { nominal bilateral }\end{array}$} & & $2,005^{\star \star}$ & & & & & $-0,808$ & 1,288 \\
\hline & & $(0,83)$ & & & & & $(0,84)$ & $(1,13)$ \\
\hline \multirow[t]{2}{*}{ Inflación promedio } & & & $1,856^{\star * \star}$ & & & & 2,098 & 4,669 \\
\hline & & & $(1,31)$ & & & & $(2,69)$ & $(2,95)$ \\
\hline \multirow[t]{2}{*}{ Volatilidad de la inflación } & & & & $4,637^{\star \star \star}$ & & & $-1,399$ & $-0,687$ \\
\hline & & & & $(1,01)$ & & & $(1,51)$ & $(1,53)$ \\
\hline \multirow[t]{2}{*}{ Indicador de globalización } & & & & &, $452^{\star \star \star}$ & & 0,156 & $-1,458^{\star \star \star}$ \\
\hline & & & & & $(0,38)$ & & $(0,11)$ & $(0,33)$ \\
\hline \multirow[t]{2}{*}{ Resistencia salarial } & & & & & & $0,059^{\star \star}$ & $0,074^{*}$ & $0,097^{\star *}$ \\
\hline & & & & & & $(0,03)$ & $(0,04)$ & $(0,04)$ \\
\hline Efectos fijos por país & No & No & No & No & No & No & No & Sí \\
\hline Efectos fijos temporales & Sí & Sí & Sí & Sí & Sí & Sí & Sí & Sí \\
\hline Observaciones & 118 & 118 & 118 & 118 & 118 & 118 & 118 & 118 \\
\hline R-cuadrado & 0,844 & 0,785 & 0,867 & 0,813 & 0,801 & 0,786 & 0,787 & 0,897 \\
\hline
\end{tabular}

Fuente: Elaboración propia.

Nota: La variable dependiente es el coeficiente de traspaso total estimado (tipo de cambio nominal bilateral con los Estados Unidos) para la ventana de tiempo $\tau$ y el país $i$. Error estándar entre paréntesis. Significación al $\left.1 \%{ }^{(* \star}\right)$, el $5 \%\left({ }^{* \star}\right)$ y el $10 \%\left(^{*}\right)$. Se excluyeron las observaciones extremas en que el coeficiente de traspaso del tipo de cambio a los precios al consumidor y el coeficiente de resistencia salarial eran superiores a 1. 